\title{
Seismic imaging of reservoir flow properties: Resolving water influx and reservoir permeability
}

\section{W. Vasco and Henk Keers*}

\begin{abstract}
Methods for geophysical model assessment, in particular the computation of model parameter resolution, indicate the value and the limitations of time-lapse data in estimating reservoir flow properties. A trajectory-based method for computing sensitivities provides an effective means to compute model parameter resolution. We examine the common situation in which water encroaches into a reservoir from below, as due to the upward movement of an oil-water contact. Using straight-forward techniques we find that, by including reflections off the top and bottom of a reservoir tens of meters thick, we can infer reservoir permeability based upon time-lapse data. We find that, for the case of water influx from below, using multiple time-lapse 'snapshots' does not necessarily improve the resolution of reservoir permeability. An application to time-lapse data from the Norne field illustrates that we can resolve the permeability near a producing well using reflections from three interfaces associated with the reservoir.

Keywords (Time-lapse geophysics, reservoir characterization, permeability estimation)
\end{abstract}

${ }^{*}$ Earth Sciences Division, Berkeley Laboratory, Berkeley, CA 94720 


\section{INTRODUCTION}

Seismic time-lapse monitoring has become an important component of field development and planning. To date, the time-lapse changes are most often used in a qualitative fashion, to identify swept areas of a reservoir and to track advancing fluid contacts (Swanston et al. 2003, MacBeth et al. 2005, Reid et al. 2005, Osdal et al. 2006). Increasingly, there are efforts to use the time-lapse data in a more quantitative way, as a basis for systematically updating a given reservoir model (Landa and Horne 1997, He et al. 1998, Huang et al. 1998, Gosselin et al. 2003, Vasco et al. 2004, Vasco 2004a, MacBeth and AlMaskeri 2006).

As one might expect, relating time-lapse amplitude changes to reservoir properties, such a permeability, is a complicated endevour depending upon many factors. Firstly, there are a host of issues associated with relating changes in seismic waveforms to changes in the condition of the reservoir (Tura and Lumley 1998, Landro 2001). Secondly, there is another set of issues which arise when trying to infer flow properties from changes in the saturations and pressures within the reservoir. The range of complexities leads one to wonder, 'How well can we infer reservoir flow properties based upon geophysical observations such as time-lapse amplitude changes?'.

Our goal in this paper is to devise a measure of the resolution of reservoir permeability provided by time-lapse seismic data. The particular approach we will take involves the computation of model parameter resolution (Aki and Richards 1980). This quantitative measure of model parameter resolution is well established in the geophysical community and was introduced some time ago (Wiggins 1972). The concept is related to the idea of resolving power, promoted by Backus and Gilbert (1968). A novel element of our approach is associated with the computation of the model parameter sensitivities which form the basis of the resolution calculations. We shall utilize an efficient trajectory-based approach for sensitivity computations, first discussed in Vasco et al. (2004)

\section{The importance of model assessment}

In previous attempts at imaging flow properties based upon seismic time-lapse amplitude changes the emphasis was on deriving specific models. To date, there has been little or no work on assessing model parameter estimates. By model parameter assessment we mean determining the quality of a solution. For example, assessment may involve estimating model parameter covariances or examining the resolution of the model parameters (Menke 1984). This is a notable oversight, particularly given the complexity of the task at hand. Time-lapse monitoring is still an emerging technology and there are some important issues which need to be examined. For example, given reflections from various interfaces within and bounding the reservoir, what data best constrain the flow properties? Can one distinguish permeability variations as a function of depth within a given reservoir? What is the value of numerous time-lapse surveys, or snapshots, in inferring permeability within the reservoir? These are some of the questions that we shall examine in this initial investigation.

\section{METHODOLOGY}

\section{The linearized inverse problem and its solution}

While the equations governing the evolution of saturation and pressure within the reservoir, the forward problem, are linear in permeability (Peaceman 1977), the inverse problem is non-linear. This is due to the fact that both the material property coefficients, porosity and permeability, as well as the fields, saturation and pressure, are unknowns. Because the field and material property variables occur as products in the equations, polynomial terms are introduced, rendering the inverse problem non-linear (Everett 1996, Vasco 2000). Additional nonlinearities are present in the relationship between time lapse seismic data and saturation and pressure changes in the reservoir. Model assessment is a difficult task for non-linear inverse problems and, unlike the situation for linear inverse problems, no completely satisfactory yet practical algorithm exists. In order to allow for the nonlinearity, and yet retain the advantages afforded by a linear inverse problem, we conduct an iterative, linearized inversion of the time-lapse data. In this approach we adopt an initial or starting model of reservoir flow properties, e.g. permeability, and successively update it to fit the observations.

The updates are based upon a linearization of the relationship between a perturbation in porosity and permeability and a perturbation in the seismic amplitude changes. For example, we denote the permeability model associated with the $n$-th iteration by $\mathbf{k}_{n}$. Similarly, we shall denote the porosity at the $n$-th iteration by $\boldsymbol{\Phi}_{n}$. We consider a discrete model, consisting of grid blocks or cells, thus the permeability field is a vector, the components of which are the grid block permeabilities. The permeability at the $n$-th iteration is a perturbation of the model obtained at the previous, $(n-1)$-st, iteration

$$
\mathbf{k}_{n}=\mathbf{k}_{n-1}+\delta \mathbf{k}_{n}
$$

and similarly for porosity. In the derivation that follows we shall relate updates in porosity and permeability to updates in saturation and pressure and ultimately to changes in seismic amplitude. That is, we shall ignore the effect of a change in porosity and strain due to compaction. This is done in order to keep the presentation simple and does not reflect a limitation of our approach. We can also include the effects of porosity changes and 
strain if necessary. An update of the porosity and permeability model, $\delta \boldsymbol{\Phi}_{n}$ and $\delta \mathbf{k}_{n}$, will produce a change in the evolution of saturation and pressure as the reservoir is produced. Correspondingly, there will be a perturbation in the time-lapse seismic waveform differences between time $T_{0}$ and $T_{1}$. We emphasize that this is a perturbation in the change in the waveforms during the $T_{1}-T_{0}$ time interval.

In what follows we shall focus on the amplitude changes associated with reflections from layers within and bounding the reservoir. Seismic reflections from the reservoir interval depend upon several different classes of variables. First, there are variables associated with the raw composition of the solid portion of the rock: the moduli and the proportion of the various constituents of the rock. Second, there are variables related to the history of the rock: the chemical and physical conditions under which the reservoir host rock evolved, this includes the nature of the cementation. Third, there are variable related to the fluid content and state of the rock: the fluid properties, the fluid saturations and distribution, the fluid pressures. Finally there are variables corresponding to the stress state of the rock, these variables are typically linked to the fluid pressures.

Because we are interested in changes associated with reservoir production and their relationship to flow properties, we shall concentrate on the relationship between the amplitudes of reflected waves and the fluid saturations (s), the fluid pressures $(\mathbf{p})$, and the porosity $(\boldsymbol{\Phi})$. We will assume that the influence of the other variables may be encapsulated in an appropriate rock physics model (Hoversten et al. 2003, Vasco et al. 2004). Thus, we consider a reflection from a particular point within the reservoir, for a particular moment in time, to be of the form $A(\mathbf{s}, \mathbf{p}, \boldsymbol{\Phi})$. Note that the seismic amplitudes depend upon the the porosity and permeability implicitly, through the saturation and pressure variables $\mathbf{s}$ and p. There is also an explicit dependence of the amplitude on porosity $\boldsymbol{\Phi}$ because the strength of a reflection also depends on the elastic moduli and porosity of the bulk rock, independent of the fluid within the pore space. The linearized relationship between perturbations in fluid saturations and pressures and the reflection amplitudes is given by a Taylor series expansion of $A(\mathbf{s}, \mathbf{p}, \mathbf{\Phi})$. To first order in the perturbations $\delta \mathbf{s}, \delta \mathbf{p}$, and $\delta \boldsymbol{\Phi}$ we have

$$
\delta A(\mathbf{s}, \mathbf{p}, \boldsymbol{\Phi})=\nabla_{\mathbf{s}} A \cdot \delta \mathbf{s}+\nabla_{\mathbf{p}} A \cdot \delta \mathbf{p}+\nabla_{\mathbf{\Phi}} A \cdot \delta \boldsymbol{\Phi} .
$$

The notation $\nabla_{\mathbf{s}} A$ signifies the partial derivatives of the amplitude function $A(\mathbf{s}, \mathbf{p}, \boldsymbol{\Phi})$. Specifically, the quantity $\nabla_{\mathbf{s}} A$ is a vector with the $j$-th component equal to

$$
\left(\nabla_{\mathbf{s}} A\right)_{j}=\frac{\partial A}{\partial s_{j}},
$$

similarly for $\nabla_{\mathbf{p}} A$ and $\nabla_{\boldsymbol{\Phi}} A$. For the following derivation we shall neglect the pressure terms and only concentrate on saturation and porosity changes. This will simplify the calculations and, as indicated below, this is an appropriate approximation for our North Sea field application. Note that this is not always the case, in many situations we must take pressure into consideration and work with the full expression (2). Neglecting the effect of pressure perturbations, the expression for the amplitude perturbation reduces to

$$
\delta A(\mathbf{s}, \mathbf{\Phi})=\nabla_{\mathbf{s}} A \cdot \delta \mathbf{s}+\nabla_{\mathbf{\Phi}} A \cdot \delta \boldsymbol{\Phi}
$$

For time-lapse amplitude data we need to consider the state of the reservoir at two distinct times $T_{0}$ and $T_{1}$. Let us denote these amplitude changes over the time interval $T_{0}$ to $T_{1}$ by an overbar, thus,

$$
\bar{A}\left(\mathbf{s}^{0}, \boldsymbol{\Phi}^{0} ; \mathbf{s}^{1}, \mathbf{\Phi}^{1}\right)=A\left(\mathbf{s}^{1}, \boldsymbol{\Phi}^{1}\right)-A\left(\mathbf{s}^{0}, \boldsymbol{\Phi}^{0}\right)
$$

where $A\left(\mathbf{s}^{0}, \boldsymbol{\Phi}^{0}\right)$ and $A\left(\mathbf{s}^{1}, \boldsymbol{\Phi}^{1}\right)$ denote the seismic amplitudes at times $T_{0}$ and $T_{1}$, respectively. We assume that the surveys have been cross-equalized such that the amplitudes and frequency content of the seismic traces are roughly equivalent (Rickett and Lumley 2001). Then, in forming (4) the porosity term will cancel for reservoirs which have not undergone compaction and the time-lapse difference takes the form

$$
\delta \bar{A}\left(\mathbf{s}^{0}, \mathbf{s}^{1}\right)=\nabla_{\mathbf{s}^{1}} A \cdot \delta \mathbf{s}^{1}-\nabla_{\mathbf{s}^{0}} A \cdot \delta \mathbf{s}^{0} .
$$

As mentioned above, the saturation evolution depends upon the permeability and porosity variations within the reservoir. As noted by Vasco et al. (2004) there is a trade-off between permeability and porosity which is difficult to resolve. In what follows we shall deal with the trade-off by neglecting the dependence on porosity. This is a reasonable approach in many situations because permeability is often the factor controlling the flow. An alternative treatment involves considering the permeability to be a known function of porosity and writing the equations soley in terms of porosity. Such an approach works well in situations in which there is a well established relationship between porosity and permeability. If the saturations are functions of the permeability variations within the reservoir, e.g. $\mathbf{s}^{0}(\mathbf{k})$ and $\mathbf{s}^{1}(\mathbf{k})$, we may write $\delta \mathbf{s}^{0}$ and $\delta \mathbf{s}^{1}$ as $\nabla_{\mathbf{k}} \mathbf{s}^{0} \cdot \delta \mathbf{k}$ and $\nabla_{\mathbf{k}} \mathbf{s}^{1} \cdot \delta \mathbf{k}$, respectively. And hence, equation (5) takes the form

$$
\delta \bar{A}\left(\mathbf{s}^{0}, \mathbf{s}^{1}\right)=\left[\nabla_{\mathbf{s}^{1}} A \cdot \nabla_{\mathbf{k}} \mathbf{s}^{1}-\nabla_{\mathbf{s}^{0}} A \cdot \nabla_{\mathbf{k}} \mathbf{s}^{0}\right] \cdot \delta \mathbf{k} .
$$

In Vasco et al. (2004) the sensitivities in equation (6) are computed in an efficient two-step fashion. First, the partial derivatives $\nabla_{\mathbf{s}^{0}} A$ and $\nabla_{\mathbf{s}^{1}} A$ are computed using numerical differencing. Second, as outlined below, the coefficients $\nabla_{\mathbf{k}} \mathbf{s}^{0}$ and $\nabla_{\mathbf{k}} \mathbf{s}^{1}$ are calculated using a trajectorybased algorithm which is similar in respects to geophysical ray methods.

We can write the expression (6) more compactly by defining the coefficient matrix

$$
\mathrm{g}=\left[\nabla_{\mathbf{s}^{1}} A \cdot \nabla_{\mathbf{k}} \mathbf{s}^{1}-\nabla_{\mathbf{s}^{0}} A \cdot \nabla_{\mathbf{k}} \mathbf{s}^{0}\right]
$$

resulting in the expression

$$
\delta \bar{A}\left(\mathbf{s}^{0}, \mathbf{s}^{1}\right)=\mathbf{g} \cdot \delta \mathbf{k} .
$$


If we assemble a suite of reflections from various points on an interface of interest, as well as from various reflectors associated with the reservoir, we may build up a system of equations

$$
\delta \overline{\mathbf{A}}=\mathbf{G} \cdot \delta \mathbf{k}
$$

where $\delta \overline{\mathbf{A}}$ is a vector of amplitude difference residuals and $\mathbf{G}$ is a matrix, known as the sensitivity matrix. The $k$-th row of $\mathbf{G}$ consists of the vector $\mathbf{g}_{k}$, the coefficients associated with the $k$-th difference.

The linear relationship between $\delta \overline{\mathbf{A}}$ and $\delta \mathbf{k}$, given by equation (9), provides the basis for the iterative updating scheme described at the beginning of this section. In particular, we can solve the linear system of equations (9) for for the update $\delta \mathbf{k}$ to a given model. Thus, given the $\mathbf{k}_{n-1}$ permeability model we compute the matrix $\mathbf{G}$ and the residuals $\delta \overline{\mathbf{A}}$ and solve equation (9) for the update $\delta \mathbf{k}_{n}$. The process is repeated until convergence to a model or an adequate reduction of the residuals.

Typically, the linearized system (9) is poorly determined with the system of equations either underdetermined, inconsistent, or both (Menke 1984). Thus, equation (9) cannot be solved in the conventional sense because the matrix $\mathbf{G}$ is ill-conditioned (Noble and Daniel 1977 , p. 170). There are a number of ways to modify the problem to remove the ill-conditioning, as discussed in Menke (1984). One of the more common approaches involves writing (9) as a penalized least squares problem. That is, rather than solve the system (9), we minimize the function

$$
L\left(\delta \mathbf{k}_{n}\right)=\left|\delta \overline{\mathbf{A}}-\mathbf{G} \cdot \delta \mathbf{k}_{n}\right|_{2}
$$

where $|\mathbf{x}|_{2}$ signifies the $l_{2}$ norm of the vector $\mathbf{x}$. The function (10) represents the sum of the squares of the residuals, and minimizing $L\left(\delta \mathbf{k}_{n}\right)$ is equivalent to solving (9) in a least squares sense.

While a least squares formulation is effective in dealing with inconsistent equations there is still the issue of non-uniqueness which occurs if the equations are under-determined. In that case, the normal equations associated with the minimization of $L\left(\delta \mathbf{k}_{n}\right)$ will be ill-conditioned (Menke 1984). Introducing penalty terms is one way to remove the non-uniqueness, an approach known as regularization (Parker 1994, p. 137). Rather than minimize $L\left(\delta \mathbf{k}_{n}\right)$ we minimize an augmented penalty function

$$
F\left(\delta \mathbf{k}_{n}\right)=\left|\delta \overline{\mathbf{A}}-\mathbf{G} \cdot \delta \mathbf{k}_{n}\right|_{2}+W_{n}\left|\delta \mathbf{k}_{n}\right|_{2}++W_{r}\left|\mathbf{D} \delta \mathbf{k}_{n}\right|_{2}
$$

where the two additional terms are included in order to minimize the size and roughness of the deviation $\delta \mathbf{k}_{n}$ at the $n$-th iteration. The scalars $W_{n}$ and $W_{r}$ are weighting factors controlling the relative importance of minimizing the perturbation size and producing a smooth model to fitting the observations. The matrix $\mathbf{D}$ is a finite difference approximation to a spatial differencing operator, the discrete equivalent of a Laplacian operator. It may be shown that the estimate, $\delta \hat{\mathbf{k}}_{n}$, minimizing the penalized misfit $F\left(\delta \mathbf{k}_{n}\right)$ may be written in the form

$$
\delta \hat{\mathbf{k}}_{n}=\mathbf{G}^{\dagger} \delta \overline{\mathbf{A}}
$$

where $\mathbf{G}^{\dagger}$ is the generalized inverse (Aki and Richards, p. 684). The exact form of the generalized inverse is

$$
\mathbf{G}^{\dagger}=\left(\boldsymbol{\Gamma}^{T} \boldsymbol{\Gamma}\right)^{-1} \boldsymbol{\Gamma}^{T}
$$

where

$$
\boldsymbol{\Gamma}=\left[\begin{array}{c}
\mathbf{G} \\
\sqrt{W_{n}} \mathbf{I} \\
\sqrt{W_{r}} \mathbf{D}
\end{array}\right]
$$

(Tarantola 1987, Pratt and Chapman 1992). We should note that the formal solution (12) is usually never explicitly formed. Rather, the normal equations, a linear system, associated with the minimization problem (11) are solve directly for $\delta \hat{\mathbf{k}}_{n}$ using a method such as the LSQR algorithm (Paige and Saunders 1982). For more details on the direct solution, readers should consult Vasco et al. (1999) and Vasco et al. (2004).

\section{Model parameter resolution and covariance}

The primary goal of this paper is to assess models of reservoir flow properties derived using time-lapse seismic observations. Our formal assessment will be based upon the calculation of the model parameter resolution matrix, as described in this sub-section. For completeness, we shall also discuss the construction of the covariance matrix, another important element of model assessment. Because model parameter resolution and covariance matrices are discussed extensively in several texts (Menke 1984, Tarantola 1987, Parker 1994) our account shall be brief.

The resolution matrix provides a measure of one's ability to recover the value of a model parameter in isolation, without trade-off with other parameter estimates. It is related to the idea of an averaging kernel, put forth by Backus and Gilbert (1968). The derivation of the model parameter resolution matrix follows quite simply by substituting the linearized data equations (9) into the equation giving the estimate, $\delta \hat{\mathbf{k}}_{n}$, equation (12). The resulting expression relates a hypothetical, 'exact' model to our model parameter estimates

$$
\delta \hat{\mathbf{k}}_{n}=\mathbf{G}^{\dagger} \mathbf{G} \cdot \delta \mathbf{k}
$$

The resolution matrix, given by

$$
\mathbf{R}=\mathbf{G}^{\dagger} \mathbf{G}
$$

represents a matrix describing how the true model is averaged to form the model parameter estimates. The $j$-th 
row of the resolution matrix consists of averaging coefficients measuring the contribution of each 'exact' model parameter to the estimate of the $j$-th parameter. In an ideal situation one would obtain the exact parameter in isolation, without any contribution from the other model parameters. In that case the resolution matrix would approach the identity matrix in form. Thus, we can use the diagonal components of the resolution matrix as a measure of our ability to isolate the model parameters. If a diagonal element approaches unity, we can resolve the corresponding model parameter in isolation from the others. Conversely, if a diagonal element is rather small in magnitude, there is considerable trade-off with one or more parameters. One advantage of the resolution matrix is that it only depends on the nature of the experiment, the geometry and the physics, and is not a function of the errors associated with the observations. This is an advantage when the nature of the errors is not well established, as can be the case for seismic waveform data.

The covariance matrix provides a measure of the uncertainty associated with model parameter estimates (Menke 1984, p. 65). For normally distributed errors, the expression for the covariance matrix follows directly from the covariance matrix associated with the data vector $\delta \overline{\mathbf{A}}$ and the linear mapping from the data vector to the vector of model parameter estimates, equation (12). The model parameter covariances, $\mathbf{C}_{\delta \hat{\mathbf{k}}_{n}}$, are given by

$$
\mathbf{C}_{\delta \hat{\mathbf{k}}_{n}}=\mathbf{G}^{\dagger} \mathbf{C}_{\delta \overline{\mathbf{A}}}\left(\mathbf{G}^{\dagger}\right)^{T}
$$

where $\mathbf{C}_{\delta \overline{\mathbf{A}}}$ is the data covariance matrix (Menke 1984, p. 65). There is a well-known trade-off between model parameter resolution and model parameter covariance (Menke 1984). There are a number of ways to approach this trade-off, but the intuitive idea is that as we try and isolate small features the uncertainty associated with our estimates increases.

\section{Sensitivity computation}

As is clear from equations (12), (13), (14), and (16), the calculation of the model parameter estimates and the corresponding model parameter resolution requires the elements of the sensitivity matrix $\mathbf{G}$. Because a reservoir model can be rather large, we need an efficient technique for calculating the elements of $\mathbf{G}$. An efficient trajectorybased approach for computing the elements of $\mathbf{G}$ was introduced by Vasco et al. (2004). The starting point for the two-step computations is the expression in equation (7). The first step involves computing the partial derivatives $\nabla_{\mathbf{s}^{0}} A$ and $\nabla_{\mathbf{s}^{1}} A$ by numerical differencing. The saturation is related to the seismic velocities via Gassmann's equations (Gassmann 1951) as described in Vasco et al. (2004). For a given reflection point we compute the reflection off an interface of the reservoir model using the method of Kennett (1983). At a given value of saturation the derivative is approximated by perturbing the saturation and recomputing the amplitude. Differencing these amplitudes and dividing by the saturation perturbation produces a numerical estimate of the derivative, a component of $\nabla_{\mathbf{s}^{0}} A$ or $\nabla_{\mathbf{s}^{1}} A$.

The quantities $\nabla_{\mathbf{k}} \mathbf{s}^{0}$ and $\nabla_{\mathbf{k}} \mathbf{s}^{1}$ are given by the trajectory-based algorithm presented in Vasco et al. (2004). For example, $\delta s^{1}{ }_{i}$ is given by

$$
\delta s^{1}{ }_{i}=s_{i}{ }^{\prime}\left(T^{1}\right) \int_{\Sigma^{1}{ }_{i}} \delta \Psi(\mathbf{x}) d r
$$

where the prime denotes the derivative with respect to time, $\Sigma_{i}^{1}$ denotes the trajectory from the $i$-th cell to a point on the initial position of the water front. The trajectory $\Sigma^{1}{ }_{i}$ depends upon the pressure field, as emphasized in Vasco (2004b). The specific dependence is given by the system of ordinary differential equations

$$
\frac{d \mathbf{X}}{d r}=-\nabla p(r)
$$

where $\mathbf{X}$ are the coordinate functions of the path $\Sigma^{1}{ }_{i}$. Intuitively, this relationship is due to the fact that the flow is down the gradient of the pressure field (Peaceman $1977)$. The quantity $\Psi(\mathbf{x})$ is the travel time of the water along the trajectory $\Sigma_{i}^{1}$ and is given by

$$
\Psi(r, t)=\frac{\phi(r)}{\kappa k(r)|\nabla p(r, t)|} .
$$

where $\kappa$ is the total mobility, $\phi(r)$ is the porosity, $k(r)$ is the permeability and $p(r, t)$ is the pressure (Vasco et al. 1999, 2004).

Typically, permeability is the controlling factor in the evolution of saturation within the reservoir. Therefore, we shall map the residuals into permeability variations. Based upon a Taylor's expansion of $\Psi(r, t)$ we can relate a perturbation in permeability to a perturbation in $\Psi(r, t)$

$$
\delta \Psi(r, t)=\frac{\partial \Psi}{\partial k} \delta k(r)
$$

by retaining terms of first-order in $\delta k(r)$. As noted in Vasco et al. (1999) and Vasco et al. (2004), we can account for the pressure perturbation as a correction term after each iteration and can also include porosity by including a porosity-permeability relationship. Combining equations (18), (20) and (21), we arrive at a relationship between perturbations in permeability and perturbations in saturation

$$
\delta s^{1}{ }_{i}=-s_{i}{ }^{\prime}\left(T^{1}\right) \int_{\Sigma^{1}{ }_{i}} \frac{\phi(r)}{\kappa k^{2}(r)|\nabla p(r, t)|} \delta k(r) d r .
$$

Thus, the sensitivities, the partial derivative of the saturation in the $i$-th block with respect to the permeability at $r$ is given by

$$
\frac{\partial s^{1}{ }_{i}}{\partial k(r)}=-s_{i}{ }^{\prime}\left(T^{1}\right) \frac{\phi(r)}{\kappa k^{2}(r)|\nabla p(r, t)|} .
$$


The sensitivities are trajectory-based, computed as line integrals over the paths $\Sigma^{1}{ }_{i}$ and $\Sigma_{i}^{0}$ in $(22)$. Note that when there is no significant variation in reservoir pressure during the time interval between the seismic surveys, the trajectories will be virtually identical.

\section{APPLICATIONS}

In this section we examine our ability to resolve reservoir permeability, given time-lapse amplitude changes.

\section{Two numerical illustrations}

A single layer model.We begin this sub-section with a single layer test model which is intended to serve as a base case. For the single layer case there is no trade-off in depth. That is, we shall only allow permeability variations within the layer of interest and fix all other parameters. The reservoir model is comprised of an 11 by 11 grid of blocks, for a total of 121 permeability values. The oil saturated (91\%) layer is 23 meters thick with a reference permeability distribution shown in Figure 1. A single horizontal producing well withdraws fluid from the layer while water intrudes from an underlying formation containing $85 \%$ water. A total of 2000 days of production is modeled using a numerical simulator. The calculated saturation and pressure changes within the reservoir interval are mapped into seismic velocity and density changes using Gassmann's equations and laboratory-derived empirical relationships (Vasco et al. 2004). The variations in seismic velocities and density due to changes in saturation and pressure are superimposed upon a one dimensional velocity model (Figure 2 ). The layer is bounded above and below by homogeneous half-spaces so the only interfaces are its upper and low boundaries. The impulse response for a vertically incidence $\mathrm{P}$-wave is shown in Figure 2. There are two distinct peaks, associated with the upper and lower layer boundaries, respectively.

First, we will concentrate on time-lapse changes associated with reflections off the top of the reservoir, the first reflection in Figure 2. We consider seismic amplitude changes occurring 1,800 days after the initiation of production. We form amplitude differences or changes by subtracting pre-production reflection amplitudes from amplitudes obtained from a survey 1,800 days later. The amplitude changes, shown in Figure $3 \mathrm{~A}$, are concentrated around the production well. The changes in amplitude are clearly asymmetric, an indication of the underlying heterogeneous permeability distribution.

The first step in the iterative inversion scheme involves computing the model parameter sensitivities, the coefficients of the matrix $\mathbf{G}$ in equation (9). In this test case the starting model is a uniform layer with a permeability of 150 milli-darcies. The sensitivities $\nabla_{\mathbf{s}^{0}} A$ and $\nabla_{\mathbf{s}^{1}} A$ are computed using numerical differencing, as noted above. The sensitivities with respect to permeability, $\nabla_{\mathbf{k}} \mathbf{s}^{0}$ and $\nabla_{\mathbf{k}} \mathbf{s}^{1}$, are calculated using the trajectorybased algorithm encapsulated in equations (22) and (23). The trajectories are determined from the pressure field, by way of equation (19). The trajectories corresponding to the reference permeability model are shown in Figure $3 \mathrm{~B}$, along with the pressure distribution. The paths plotted in Figure 3B are projections onto the horizontal plane of the reservoir model. The actual paths have a significant vertical component as they extend from the water saturated layer to the center of each grid block of the reservoir layer.

The total sensitivity is given by a combination of the amplitude sensitivities $\nabla_{\mathbf{s}^{0}} A$ and $\nabla_{\mathbf{s}^{1}} A$ and the saturation sensitivities $\nabla_{\mathbf{k}} \mathbf{s}^{0}$ and $\nabla_{\mathbf{k}} \mathbf{s}^{1}$, as expressed in equation ( 7 ). In Figure 4 we have plotted the amplitude sensitivities $\nabla_{\mathbf{s}^{0}} A$ in the panel on the left and the total sensitivities $\left[\nabla_{\mathbf{s}^{1}} A \cdot \nabla_{\mathbf{k}} \mathbf{s}^{1}-\nabla_{\mathbf{s}^{0}} A \cdot \nabla_{\mathbf{k}} \mathbf{s}^{0}\right]$ in the right panel. The total sensitivity is largest at some distance from the producing well, due to the differences in the rate of change in the saturation for those grid blocks.

Given the sensitivities, we can form the generalized inverse (13) and ultimately, the resolution matrix (16). The generalized inverse depends upon the weighting factors $W_{n}$ and $W_{r}$ in the penalty function (11). We chose these weights by trial and error, conducting a number of inversions with different values for $W_{n}$ and $W_{r}$. Both fitting the observations and obtaining a smooth model were considerations in adjusting $W_{n}$ and $W_{r}$. Based upon the test inversions our preferred values were $W_{n}=3.0$ and $W_{r}=3.0$. The resulting diagonal elements of the resolution matrix are shown in Figure 5 . In Figure 5 we have plotted the value of each element of the diagonal of the resolution matrix in the location of the associated cell. Recall that values of the diagonal elements close to 1 signify well resolved parameters with little or not trade-off with other parameters. As is evident from Figure 5, most elements of the resolution matrix are larger the 0.7 , the only exception is at the edges of the model. Thus, the permeabilities are well resolved, for the most part, and we can recover individual parameter values.

The favorable resolution is born out in practice when we conduct an inversion of the synthetic time-lapse amplitude changes. In Figure 6 we present the root-meansquared misfit as a function of the number of steps of our iterative inversion algorithm. It is clear that the misfit is reduced by over an order of magnitude in the ten iterations of the algorithm. Also, there is a notable reduction in scatter in the time-lapse amplitude residuals. The final permeability model does a good job of recovering the large-scale permeability variations within the layer (Figure 1) Thus, time-lapse amplitude data are useful in imaging permeability in the vicinity of a producing well. In summary, it appears that time-lapse seismic amplitude changes can be used to image permeability variations for a single layer reservoir model. 
A multilayered model. - Next we consider the case in which there are additional layers in the reservoir model. In this illustration the reservoir is composed of three layers. The lowermost layer is entirely water saturated and represents the water column. The two upper layers are oil saturation and comprise the oil column in the reservoir. A horizontal producing well in the top layer withdraws fluid and induces flow and the migration of the water from the bottom layer. The saturation changes in the two uppermost layers are shown in Figure 7 . In this test case we shall use reflections from the top of the various layers to constrain the permeability within the two uppermost layers. We will emphasize the value of the various sets of reflections, reflections from each layer, in resolving the flow properties in the two top layers. Because there is no saturation change in the lowermost layer we are unable to resolve its flow properties.

The top two layers of the reservoir model are 50.0 meters thick and contain the reference heterogeneity shown in Figure 1. The bottom layer is also 50.0 meters thick but has a uniform permeability of 150 milli-darcies. As in the one-layer model, each layer is composed of an 11 by 11 grid of cells, a total of 121 grid-blocks in each layer. A horizontal well is withdrawing fluid at a rate of 100 barrels per hour from the top-most layer. The pressure response to production is shown in Figure 8 for the top two layers. In addition, we plot the flow paths in each layer, projected into the horizontal plane of the layer. For each trajectory in Figure 8 there is a significant vertical component as each path travels from the grid block center of each layer to some point in the lower-most layer. Note that the trajectories control the sensitivity of the saturation change to permeability, as noted above [see equations (18) and (22)].

A numerical simulator was used to model a total of 2000 days of production. We shall examine the amplitude changes associated with changes in the state of the reservoir between the first month of production and 1800 days layer. Because the pressure equilibrated rapidly, the amplitude changes were dominated by the evolution of saturation within the reservoir. The seismic response is dominated by two interfaces, as indicated in Figure 9. The dominant reflection is from the top of the reservoir where there is a jump in seismic velocities and density. There is a small jump in $\mathrm{P}$ velocity between layer one and layer two of the model. The final discontinuity is between layer two and layer three, which we signify as the base of the reservoir. Thus, the seismic response is primarily composed of a top reflection and a reflection off the base of layer two. Note that the reflection off the layer 1 - layer 2 interface is not evident in Figure 9. Due to the small contrast in seismic properties between these layers and the fact that the layers are thin, the reflection is not visible in the seismic response. Thus, we shall assume that it is not possible to pick a reflection event associated with this interface.

The amplitude difference, due to changes in the saturation and pressure variations, were computed us- ing Gassmann's equations and the method of Kennett (1983). Because the pressure did not change significantly, the amplitude changes were primarily due to saturation variations. The amplitude changes associated with the top and base reflections are shown in Figure 10 . Note the asymmetric pattern due to the heterogeneous permeability distribution with changes extending to the north and west where the permeability is higher. The amplitude changes in Figure 10 form our basic data set with which to infer the permeability distribution in the top two layers. For this study we are interested in our ability to resolve permeability within the reservoir from the amplitude data. To this end, we shall compute selected elements of the model parameter resolution matrix, given in equation (16).

An important part of our resolution calculations involves computing the sensitivity matrix $\mathbf{G}$, whose rows are given in equation (7). We use the twostep, trajectory-based methodology in which the partial derivatives $\nabla_{\mathbf{s}^{0}} A$ and $\nabla_{\mathbf{s}^{1}} A$ are computed using numerical differencing. These derivatives are plotted in Figure 11 , for both layers and for both reflections. Note that the top reflection is dominated by changes in the upper-most layer, as one would expect. Similarly, the base reflection is primarily influenced by changes in layer two. The sign of the sensitivities is reversed for these two reflections because the first reflection is off the top of layer one and the second or 'base' reflection is off the base of layer two (the top of layer three). The sensitivities are relatively symmetric because they are computed with respect to the starting model which is a uniform permeability model with a constant permeability of 150 milli-darcies.

The partial derivatives $\nabla_{\mathbf{s}^{0}} A$ and $\nabla_{\mathbf{s}^{1}} A$ are combined with the trajectory-based sensitivities $\nabla_{\mathbf{k}} \mathbf{s}^{0}$ and $\nabla_{\mathbf{k}} \mathbf{s}^{1}$ to produce the rows of the sensitivity matrix associated with the $l$-th reflection

$$
\mathrm{g}_{l}=\left[\nabla_{\mathbf{s}^{1}} A_{l} \cdot \nabla_{\mathbf{k}} \mathbf{s}^{1}-\nabla_{\mathbf{s}^{0}} A_{l} \cdot \nabla_{\mathbf{k}} \mathbf{s}^{0}\right] .
$$

The rows of the sensitivity matrix associated with the top and base reflections are shown in Figure 12. Again, the sensitivities for the two reflections are of opposite signs. Furthermore, for both reflections the sensitivities are greatest in layer 2. The greater sensitivity associated with layer two is due to the fact that the influx of water must traverse layer two in order to reach layer one. Thus, all paths from layer one must pass through layer two. So layer two will be crossed by two sets of paths, those from layer one and those originating within layer two itself. The two sets of paths through the second layer mean that its coverage is greater. In addition, as is evident in Figure 8, the pressure gradient is smaller in the second layer because it is farther from the producing well. Hence, the sensitivities associated with layer two are larger because they are proportional to the inverse of the magnitude of the pressure gradient [see equation (23)]. Physically, the fluid spends a longer time in the layer with the lower pressure gradient so a change in permeability there will have a greater influence on its travel time. 
The resolution matrix is formed according to equations (13), (14), and (16), where we have taken $W_{n}=5.0$ and $W_{r}=5.0$. The diagonal elements of the resolution matrix are shown in Figure 13 for two distinct cases. The top panels in Figure 13 display the resolution of permeability within the two layers of the reservoir model for the situation in which only the top reflection is used in the inversion. The bottom panels present the case in which only the base reflection is used to constrain the permeability estimates. In both cases we obtain moderate resolution in the lowermost layer, values of around 0.5 and below. The resolution associated with the uppermost layer is rather low, 0.25 and lower.

The resolution improves significantly if we combine the top and base reflection data sets (Figure 14). By considering the two data sets together we improve the resolution by $50 \%$, with peak values of over 0.75 for the lowermost layer. We can achieve moderate resolution in the uppermost layer, peak values of around 0.5 (Figure 14). The moderate resolution estimates associated with the two data sets are confirmed by a test inversion (Figure 15). A linearized iterative inversion recovers the large-scale pattern of heterogeneity, with lower permeability to the south and higher permeability to the north.

We considered other possible combinations of seismic data and examined the associated model parameter resolution. For example, we looked at the change in resolution when we used a window to compute the amplitude change associated with a given reflection. That is, rather than consider the amplitude change associated with the peak, the amplitude changes are computed in a ten sample window by computing the RMS amplitude change. The window center is defined with respect to the peak amplitude. We found that the resolution was virtually identical for either definition of the amplitude change. We also tried including other amplitude values in addition to the top and base reflections. For example, we included the amplitude change associated with an intermediate point, located mid-way between the two reflections. We found that including these amplitude data did not change the model parameter resolution. The reservoir we modeled was rather thin, our total reservoir thickness was only about 100 meters. Thus, intermediate amplitude data may not provide independent data constraints. For a thicker reservoir, additional reflections and amplitude data may indeed improve the resolution. We also examined the value of additional time-lapse 'snapshots' in resolving reservoir permeability. In particular, we combined amplitude changes from 30 days of production to 350,1100 , and 1820 days of production (Figure 16). The amplitude changes were associated with a reflection of the top of the reservoir, similar to the left panel of Figure 10. We found that the resolution for the combined data set (Figure 17) did not differ substantially from the resolution associated with a single set of time-lapse data (top panel of Figure 13).

\section{Application at the Norne field}

The Norne field is a Norwegian Sea reservoir defined by a 9 by $3 \mathrm{~km}$ horst block of Lower and Middle Jurassic age sandstones (Khazanehdari et al. 2004, Osdal et al. 2006). The sandstones are of high quality, with porosities of 25 $30 \%$ and permeabilities varying between 200 and 2000 $\mathrm{mD}$. The field has been producing oil since 1997 and gas since 2001 from 12 wells operating from a floating production, storage, and off-loading vessel (FPSO) moored in the middle of the field. Pressure support is provided by water injection through seven wells into the oil zone and gas injection into the gas cap through one well.

The portion of the reservoir discussed here consists of the Ile, Tofte, and Tilje formations (Figure 18). The Ile and Tofte formations form the primary oil reservoir $(110 \mathrm{~m})$ while the oil-water contact generally follows the Tofte-Tilje interface (Osdal et al. 2006). There is also an overlying gas cap in the Garn formation, with a thickness of roughly $75 \mathrm{~m}$. In the region under study the main signal is thought to be induced by the movement of the oilwater contact upward through the Tofte formation and perhaps the Ile formation.

The initial seismic exploration was a large-scale regional survey shot in 1992 using a dual source and three streamers (Osdal et al. 2006). Subsequently, three high-quality Q-marine system surveys were conducted by WesternGeco in 2001, 2003, and 2004 (Ostal et al. 2006). The Q-surveys use steered streamers, reducing streamer feathering and dramatically improving repeatability. In addition, a comprehensive positioning system, calibrated marine sources, and single-sensor recording provide for high signal-to-noise and reduced system noise. All Norne seismic survey data went through the same processing sequence at WesternGeco (Ostal et al. 2006). The data were heavily contaminated by diffraction multiples and a 2D surface-related multiple elimination (SRME) was found to be the best method for their removal. In addition, Kirchoff prestack migration was applied to each survey. We utilize the time-lapse changes which occurred between the 2001 and 2003 seismic surveys. The amplitude changes associated with reflections from the top of the Ile, Tofte, and Tilje formations will constitute our data set. The amplitude changes are computed over a 8 ms time window defined with respect to the time horizon of each reflection (Figure 18).

The reservoir and seismic modeling were confined to the region surrounding a production well intersecting the Ile formation (Figure 18). The reservoir model consisted of the three formations (Ile, Tofte, and Tilje) with each layer sub-divided into a 20 by 20 grid of cells. A variable thickness was used for each layer, based upon the stacking velocity model and the two-way-traveltime estimates for the formations (Figure 18). Because our primary focus was on estimating the resolution of the reservoir permeability, the modeling was unsophisticated. For example, an averaged, uniform permeability, $600 \mathrm{mD}$, was used for 
the initial value in each layer. A single producing well, located in the Ile formation, withdraws fluid from the oil column of the reservoir. Due to the production, water from the Tilje formation encroaches into the Tofte and Ile formations. During the two year interval 2001 to 2003 the reservoir pressure did not change significantly and the main signal was due to the movement of the oil-water contact. The rock physics model used to relate saturation and pressure changes to changes in seismic attributes was a high porosity sand model used in Vasco et al. (2004). Such a model should be appropriate for the high porosity, high permeability sands comprising the Norne reservoir.

Using the techniques presented in the methodology section we computed the rows of the sensitivity matrix $(\mathrm{g})$, equation (7). We computed sensitivities for reflections off the top of the Ile, Tofte, and Tilje formations. In Figure 19 we show the sensitivities for reflections off the top and base of the Tofte formation. As in the synthetic computations, the peak sensitivities form a ring surrounding the production well. Because the well is vertical, the sensitivities form a more circular pattern. The pattern is not centered symmetrically about the production well, perhaps due to the variable reservoir thickness in the model.

Using the sensitivity matrix coefficients $\mathbf{G}$ we computed the resolution matrix $\mathbf{R}$ using equation (16). Regularization weighting factors of $W_{n}=1.3$ and $W_{r}=1.3$ were based upon test inversions, balancing the fit to the data against the model size and roughness, The diagonal elements of the resolution matrix associated with the Tofte formation are shown in Figure 20. As in the synthetic tests, the resolution is rather high for the region surrounding the well, with peak values near 0.8 , indicating that we can imaging permeability there. Thus, using the three sets of time-lapse amplitude changes (Ile, Tofte, and Tilje) we can infer permeability around the producing well.

Our final task is to use the time-lapse amplitude changes to estimate permeability variations near the producer. The relative amplitude changes are shown in Figure 21 for the three interfaces. All panels in this figure are to the same scale, an amplitude change of 200 counts. Note the largest amplitude changes are associated with reflections off the top of the Tofte and Tilje formations. Furthermore, the reflections off the Tilje formation are similar in pattern from those off the Tofte but of opposite sign. This is compatible with a saturation change within the Tofte formation with a peak change to the north and east of the well. An iterative linearized inversion of the amplitude changes produced the permeability model shown in Figure 22. In the well resolved regions the permeability is substantially lower than the $600 \mathrm{mD}$ starting values.

\section{CONCLUSIONS}

There is increasing use of time-lapse data as a means for updating reservoir models. In this paper we examined the usefulness of time-lapse seismic amplitudes in constraining reservoir permeability. The situation we examined, water influx into a formation from below due to the movement of an oil-water contact, is quite common in reservoir production. The results of this study suggest that, under favorable conditions, time-lapse seismic data can be used to image flow properties in a reservoir. However, attention must be paid to the reliability of permeability estimates and a model assessment is essential. In this paper we have focused on estimating model parameter resolution because it is, in a sense, the simplest measure of model reliability. Specifically, its construction only depends on the nature of the experiment, the geometry and underlying physics, not on the errors in the data. Thus, it is somewhat simpler to construct than model parameter covariance because errors on waveforms are not necessarily easy to estimate. The trajectory-based approach for computing model parameter sensitivities, first presented in Vasco et al. (2004), is well suited for the resolution calculations.

Our numerical tests indicated that by including reflections from the top and base of a reservoir tens of meters thick we can resolve the average permeability within the reservoir. Including internal reflections will not necessarily improve the resolution in a thin reservoir but may be of use for reservoirs which are thicker. We also found that including multiple time-lapse 'snapshots' did not appear to improve the resolution of permeability in the case of water influx from below. For the situation in which water is migrating from depth towards a shallower producer we find that the lowermost portion of the reservoir is better resolved.

\section{ACKNOWLEDGMENTS}

This work was supported by Schlumberger Oilfield Services and by the Assistant Secretary, Office of Basic Energy Sciences of the U. S. Department of Energy under contract DE-AC03-76SF00098. All computations were carried out at the Center for Computational Seismology, Berkeley Laboratory. 


\section{REFERENCES}

Aki, K. and Richards, P. G., 1980, Quantitative Seismology: W. H. Freeman and Company, San Francisco.

Backus, G. E., and Gilbert, J. F., 1968, The resolving power of gross earth data, Geophy. J. Roy. Astron. Soc., 16, 169-205.

Calvert, R., 2005, Insights and Methods for 4D Reservoir Monitoring and Characterization:, EAGE/SEG Distinguished instructor short course, No. 8, Society of Exploration Geophysicists, Tulsa.

Everett, M. E., 1996, Homotopy, polynomial equations, gross boundary data, and small Helmholtz systems: J. Comp. Phys., 124, 431-441.

Gassmann, F., 1951, Elastic waves through a packing of spheres: Geophysics, 16, 673-685.

Gosselin, O., Aanonsen, S. I., Aavatsmark, I., Cominelli, A., Gonard, R., Kolasinski, M., Ferdinandi, F., Kovacic, L., and Neylon, K., 2003, History matching using time-lapse seismic (HUTS), SPE Paper, 84464.

He, W., Guerin, G., Anderson, R. N., and Mello, U. T., 1998, Time-dependent reservoir characterization of the LF sand in the South Eugene Island 330 Field, Gulf of Mexico: The Leading Edge, 17, 1434-1438.

Hoversten, G. M., Gritto, R., Washbourne, J., and Daley, T., 2003, Pressure and fluid saturation prediction in a multicomponent reservoir using combined seismic and electromagnetic imaging: Geophysics, 68, 1580-1591.

Huang, X., Meister, L., and Workman, R., 1998, Improving production history matching using time-lapse seismic data: The Leading Edge, 17, 1430-1433.

Kennett, B. L. N., 1983, Seismic Wave Propagation in Stratified Media: Cambridge University Press.

Khazanehdari, J., Toto, R., Curtis, T., Ozdemir, H., Murineddu, A., Gehenn, J. M., and Yi, T., 2004, Quantitative analysis on the first Q-on-Q 4D programme: First Break, 22, 67-71.

Landa, J. L., and Horne, R. N., 1997, A procedure to integrate well test data, reservoir performance history, and 4-D seismic information into a reservoir description: paper SPE 38653 presented at the 1997 SPE Annual Technical Conference and Exhibition, San Antonio, Texas, October 5-8.

Landro, M., 2001, Discrimination between pressure and fluid saturation changes from time-lapse seismic data: Geophysics, 66, 836-844.

MacBeth, C., and Al-Maskeri, Y., 2006, Extraction of permeability from time-lapse seismic data: Geophysical Prospecting, 54, 333-349.
MacBeth, C., Stephen, K. D., McInally, A., 2005, The 4D signature of oil-water contact movement due to natural production in a stacked turbidite reservoir: Geophysical Prospecting, 53, 183-203.

Menke, W., 1984, Geophysical Data Analysis: Discrete Inverse Theory: Academic Press Inc., Orlando.

Noble, B., and Daniel, J. W., 1977, Applied Linear Algebra: Prentice-Hall Inc., Englewood Cliffs, N. J.

Osdal, B., Husby, O., Aronsen, H. A., Chen, N., and Alsos, T., 2006, Mapping the fluid front and pressure buildup using 4D data on Norne Field, The Leading Edge, 25, 1134-1141.

Paige, C. C., and Saunders, M. A., 1982, LSQR: An algorithm for sparse linear equations and sparse linear systems: ACM Trans. Math. Software, 8, 195-209.

Parker, R. L., 1994, Geophysical Inverse Theory: Princeton University Press, Princeton, N. J.

Peaceman, D. W., 1977, Fundamentals of Numerical Reservoir Simulation: Elsevier Scientific Publishing.

Pratt, R. G., and Chapman, C. H., 1992. Traveltime tomography in anisotropic media-II. Application. Geophys. J. Int., 109, 20-37.

Reid, F. J. L., Bertrand, A., McInally, A. T., and MacBeth, C., 2005, 4D signal enhancement using singularvalue decomposition: application to mapping oil-water contact movement across the Nelson Field, Geophysical Prospecting, 53, 253-263.

Rickett, J. E., and Lumley, D. E., 2001, Crossequalization data processing for time-lapse seismic reservoir monitoring: A case study from the Gulf of Mexico: Geophysics, 66, 1015-1025.

Swanston, A. M., Flemings, P. B., Comisky, J. T., and Best, K. D., 2003, Time-lapse imaging at Bullwinkle field, Green Canyon 65, offshore Gulf of Mexico, Geophysics, 68, 1470-1484.

Tarantola, A.., 1987. Inverse Problem Theory: Methods for Data Fitting. Elsevier Science Publishers, Amsterdam.

Tura, A., and Lumley, D. E., 1998, Subsurface fluid-flow properties from time-lapse elastic-wave reflection data: SPIE Proceedings, 43rd Annual Meeting, 125-138.

Vasco, D. W., 2004a, Seismic imaging of reservoir properties: Time-lapse pressure changes: Geophysics 69, $511-521$.

Vasco, D. W., 2004b, An asymptotic solution for twophase flow in the presence of capillary forces, Water Resources Research 40, 1-13. 
Vasco, D. W., 2000, An algebraic formulation of geophysical inverse problems, Geophys. J. Int. 142, 970-990.

Vasco, D. W., Datta-Gupta, A., Behrens, R., Condon, P., and Rickett, J., 2004, Seismic imaging of reservoir properties: Time-lapse amplitude changes: Geophysics $69,1425-1442$.

Vasco, D. W., Yoon, S. and Datta-Gupta, A., 1999. Integrating dynamic data into high-resolution reservoir models using streamline-based analytic sensitivity coefficients: SPE Jour., 4, 389-399.

Wiggins, R. A., 1972, The general linear inverse problem: Implications of surface waves and free oscillations for Earth structure, Review of Geophysics and Space Physics 10, 251-285.

\section{FIGURE CAPTIONS}

Figure 1. (Left Panel) Reference permeability model used to compute synthetic time-lapse saturation and pressure changes. (Right Panel) Permeability estimates derived using an iterative linearized inversion technique.

Figure 2. (Left Panel) Depth variation of seismic velocities and density for the reservoir interval. (Right Panel) Seismic impulse response associated with the velocity and density model. The reflections are off the top and the bottom of the reservoir layer.

Figure 3. (A) Time-lapse amplitude change, in percent, between 1 day and 1800 days of production. (B) Pressure variations within the reservoir. The curves in this figure denote flow lines within the reservoir.

Figure 4. (Left Panel) Time-lapse amplitude sensitivities, $\nabla_{\mathbf{s}^{0}} A$. (Right Panel) Total permeability sensitivity $\mathrm{g}=\left[\nabla_{\mathbf{s}^{1}} A \cdot \nabla_{\mathbf{k}} \mathbf{s}^{1}-\nabla_{\mathbf{s}^{0}} A \cdot \nabla_{\mathbf{k}} \mathbf{s}^{0}\right]$.

Figure 5. Diagonal elements of the model parameter resolution matrix $\mathbf{R}$.

Figure 6. (Left Panel) Sum of the squares of the residuals as a function of the number of iterations. (Right Panel) Misfit computed using the initial permeability model (open circles) and the final permeability model (filled squares).

Figure 7. (Left Panel) Saturation change in the top layer of the reservoir model. (Right Panel) Saturation change in the bottom layer of the reservoir model.

Figure 8. (Left Panel) Pressure variations within the top layer of the reservoir. (Right Panel) Pressure variations within the bottom layer of the reservoir. The curves in this figure denote flow lines within the reservoir.

Figure 9. (Left Panel) Depth variation of seismic velocities and density for the reservoir interval. (Right Panel) Seismic impulse response for the seismic model. The reflections are associated with the top and bottom of the reservoir model.

Figure 10. (Left Panel) Time-lapse amplitude change for the top reflector, in percent, between 1 day and 1800 days of production. (Right Panel) Time-lapse amplitude change for the base reflector, in percent, between 1 day and 1800 days of production.

Figure 11. (Top Panels) Time-lapse amplitude sensitivities for the top reflection. (Bottom Panels) Timelapse amplitude sensitivities for the base reflection.

Figure 12. (Top Panels) Total sensitivities for the top reflection. (Bottom Panels) Total sensitivities for the base reflection.

Figure 13. (Top Panels) Diagonal elements of the resolution matrix for the top reflection. (Bottom Panels) Diagonal elements of the resolution matrix for the base reflection.

Figure 14. Diagonal elements of the resolution matrix for the top and base reflections.

Figure 15. Model parameter estimates from the iterative linearized inversion algorithm. 
Figure 16. Time-lapse amplitude changes for three different time-intervals, 360, 1100, and 1820 days.

Figure 17. Diagonal elements of the resolution matrix for the case in which three time-lapse 'snapshots' are used.

Figure 18. Two-way-traveltime to the three primary formations in the Norne reservoir.

Figure 19. Model parameter sensitivities associated with the top reflection (top) and the bottom reflection (bottom) of the Tofte formation.

Figure 20. Diagonal elements of the resolution matrix for the Tofte formation.

Figure 21. Time-lapse amplitude changes for the three primary formations at the Norne field.

Figure 22. Result of an iterative linearized inversion of the time-lapse amplitude changes at the Norne field. 

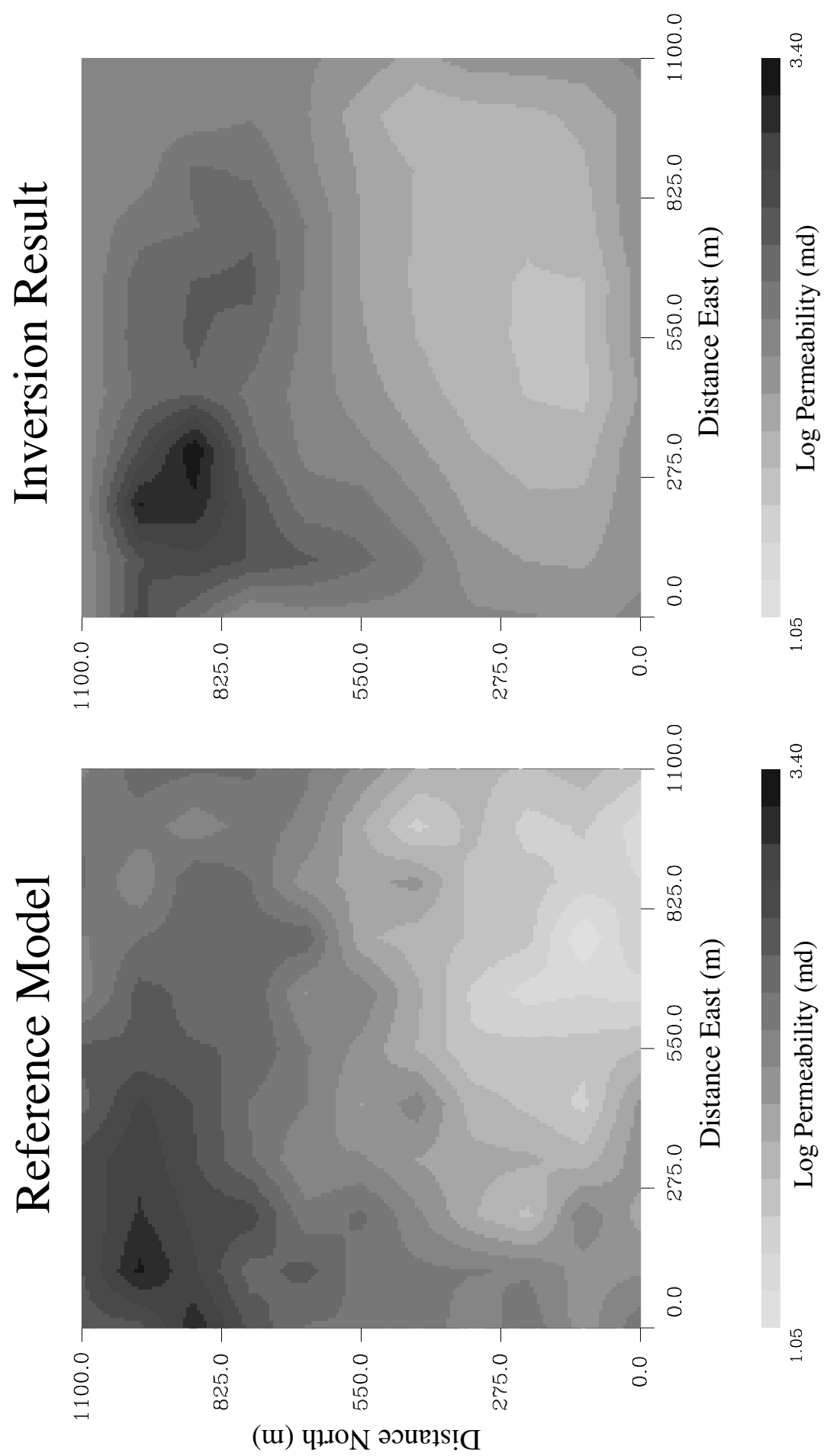

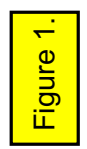



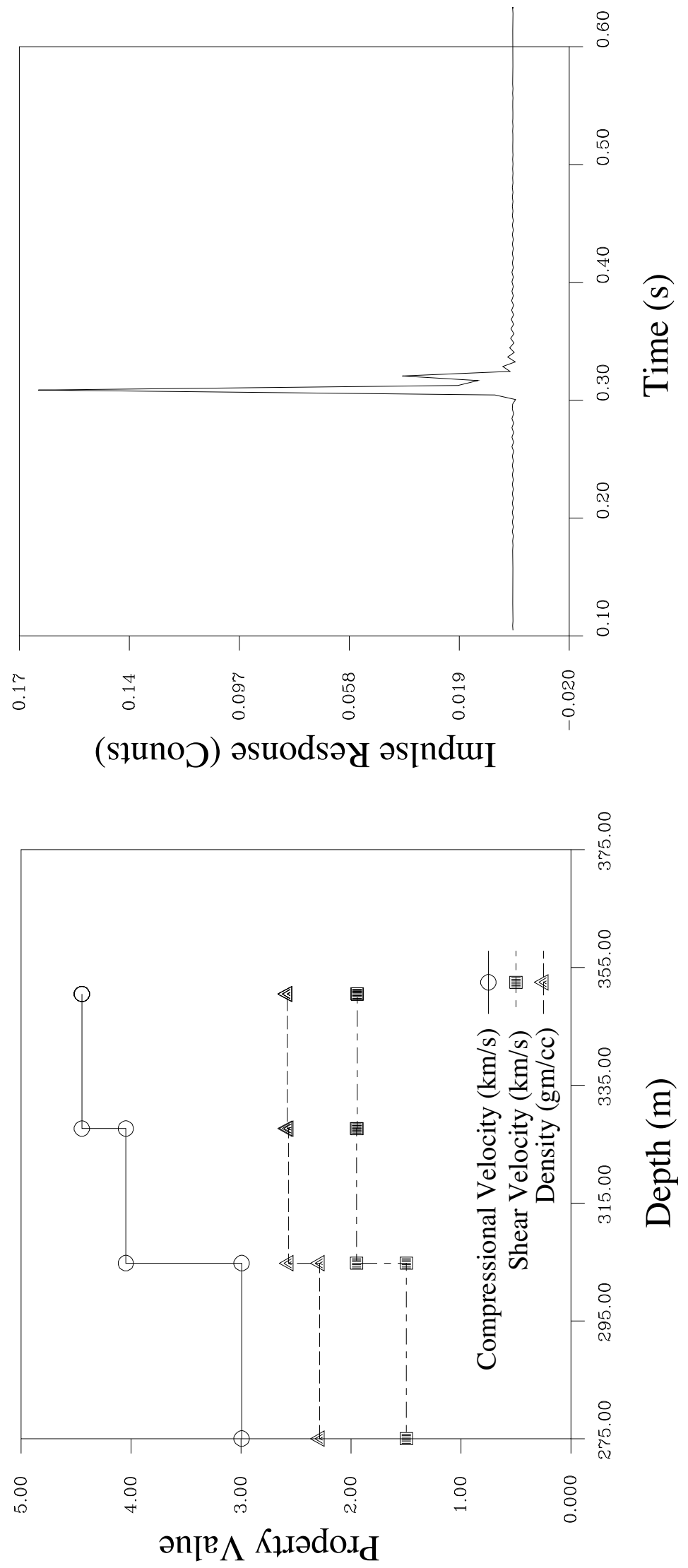


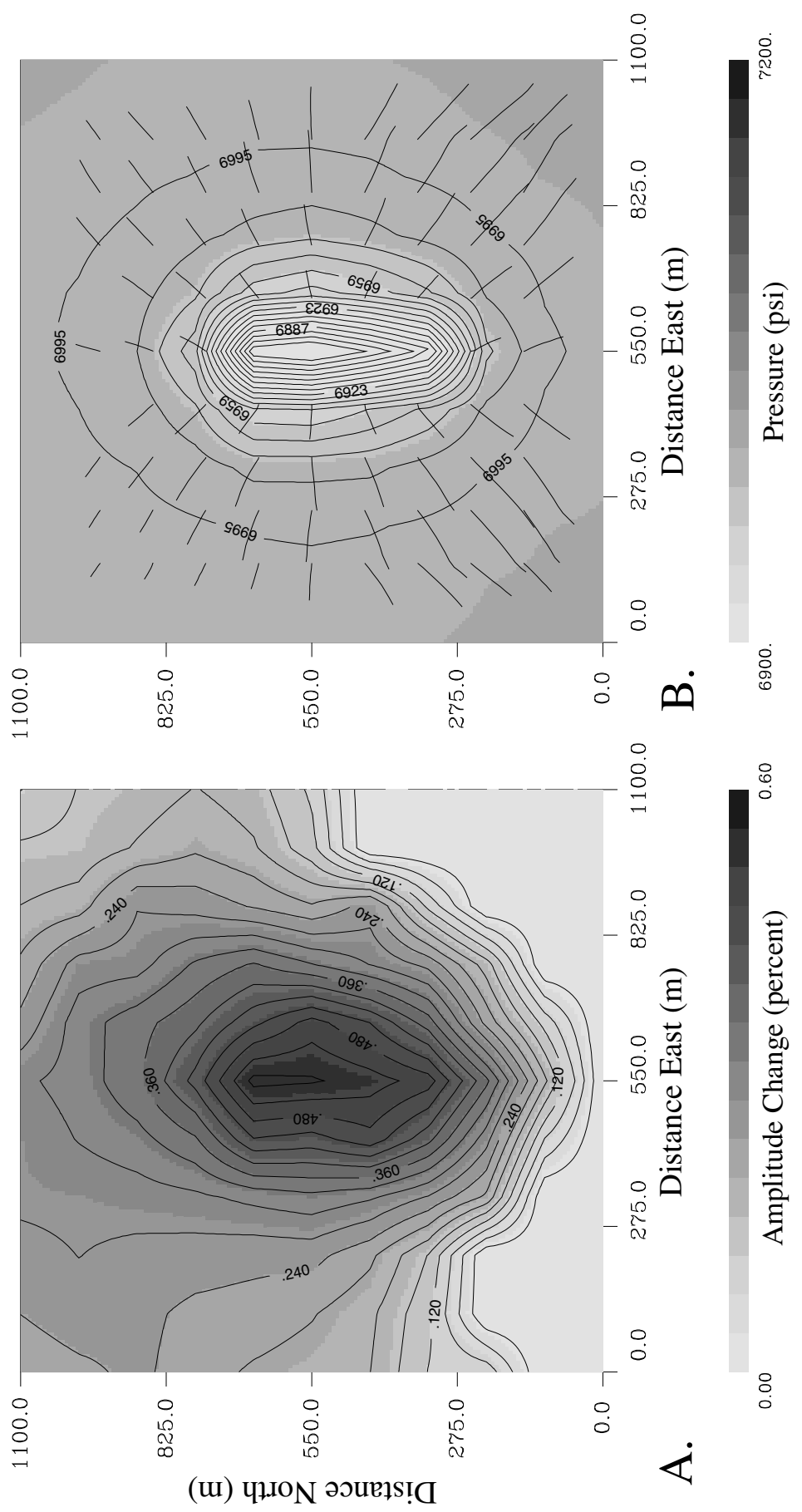

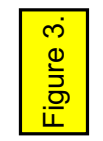




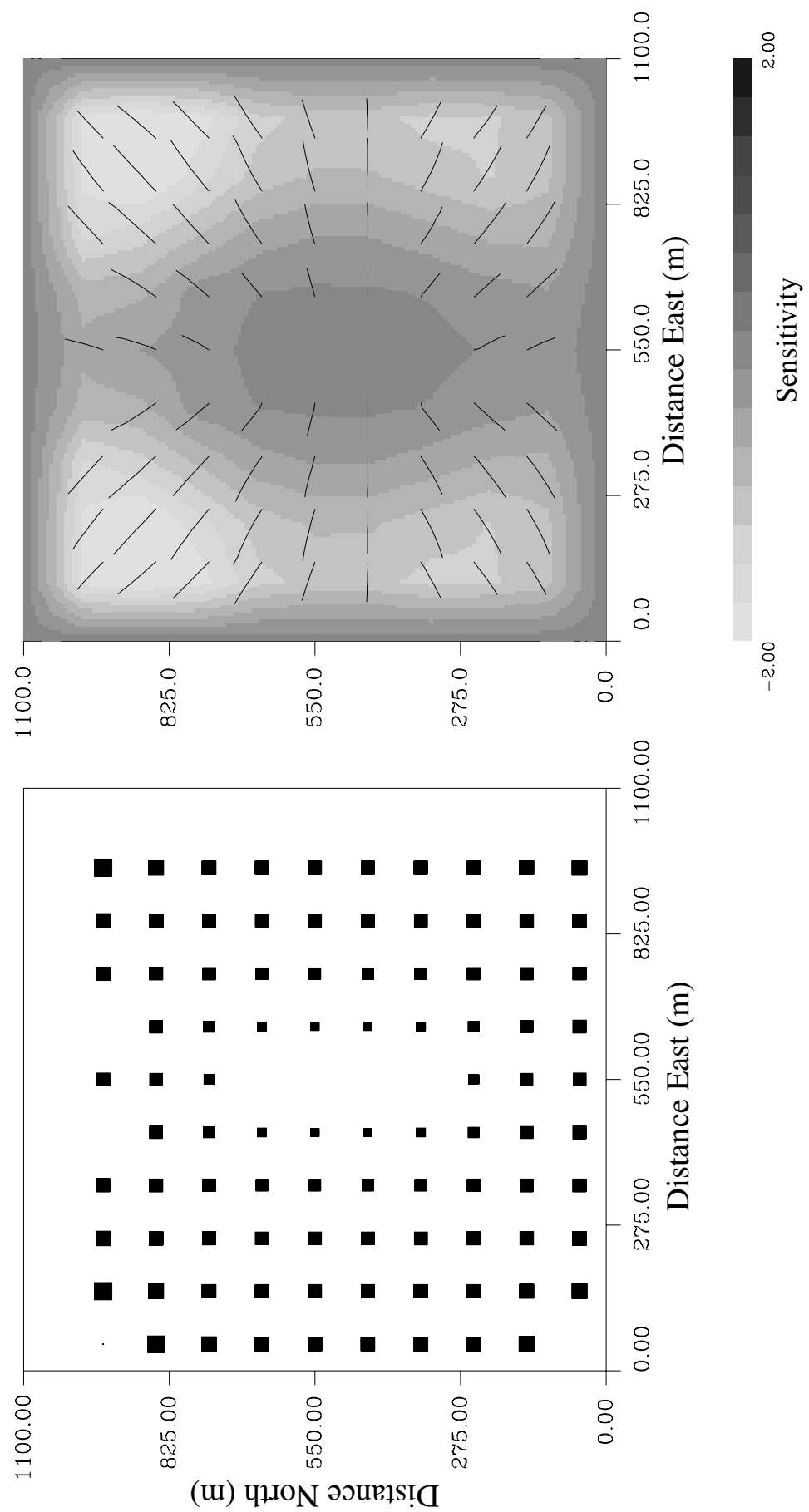




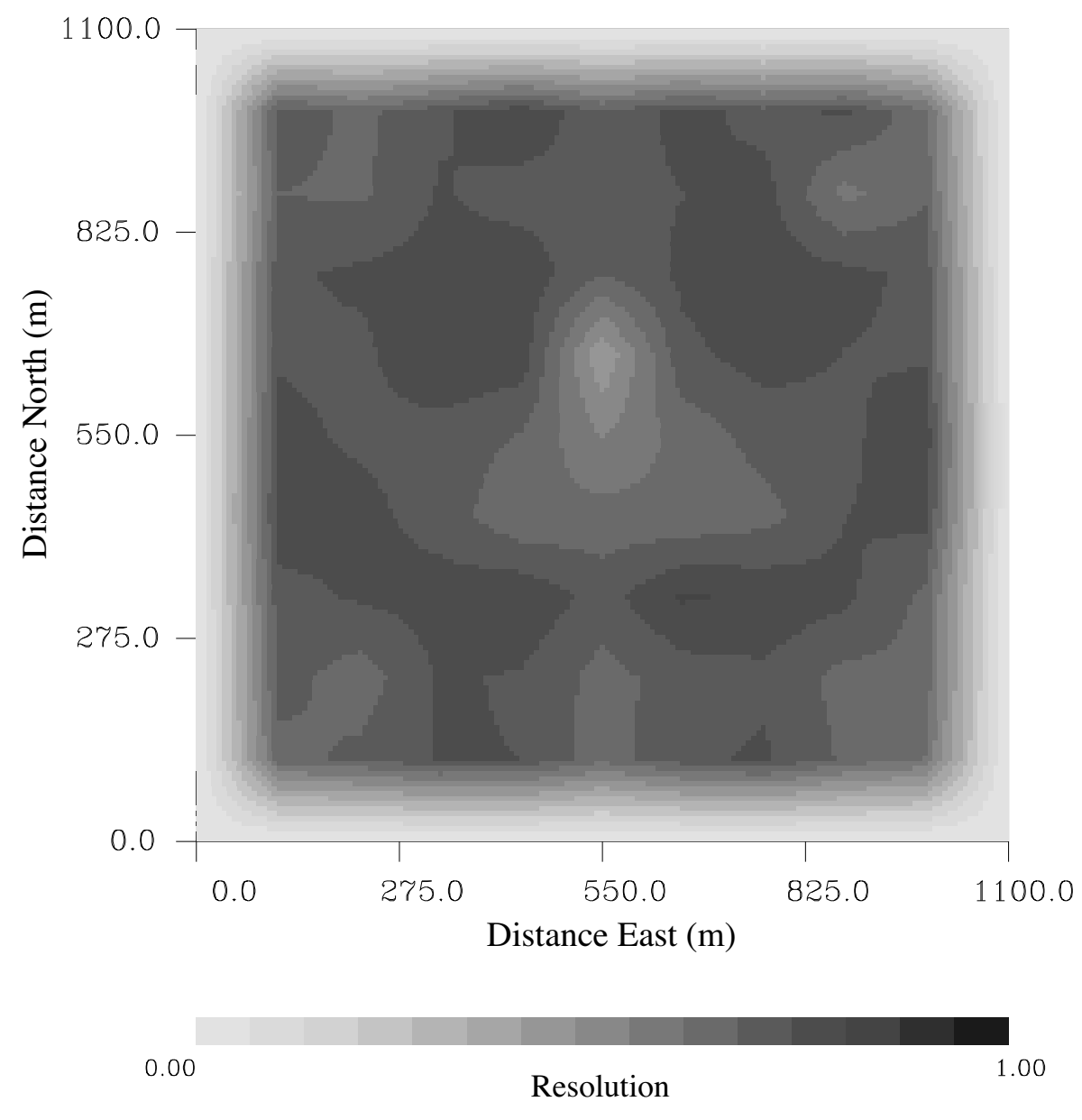



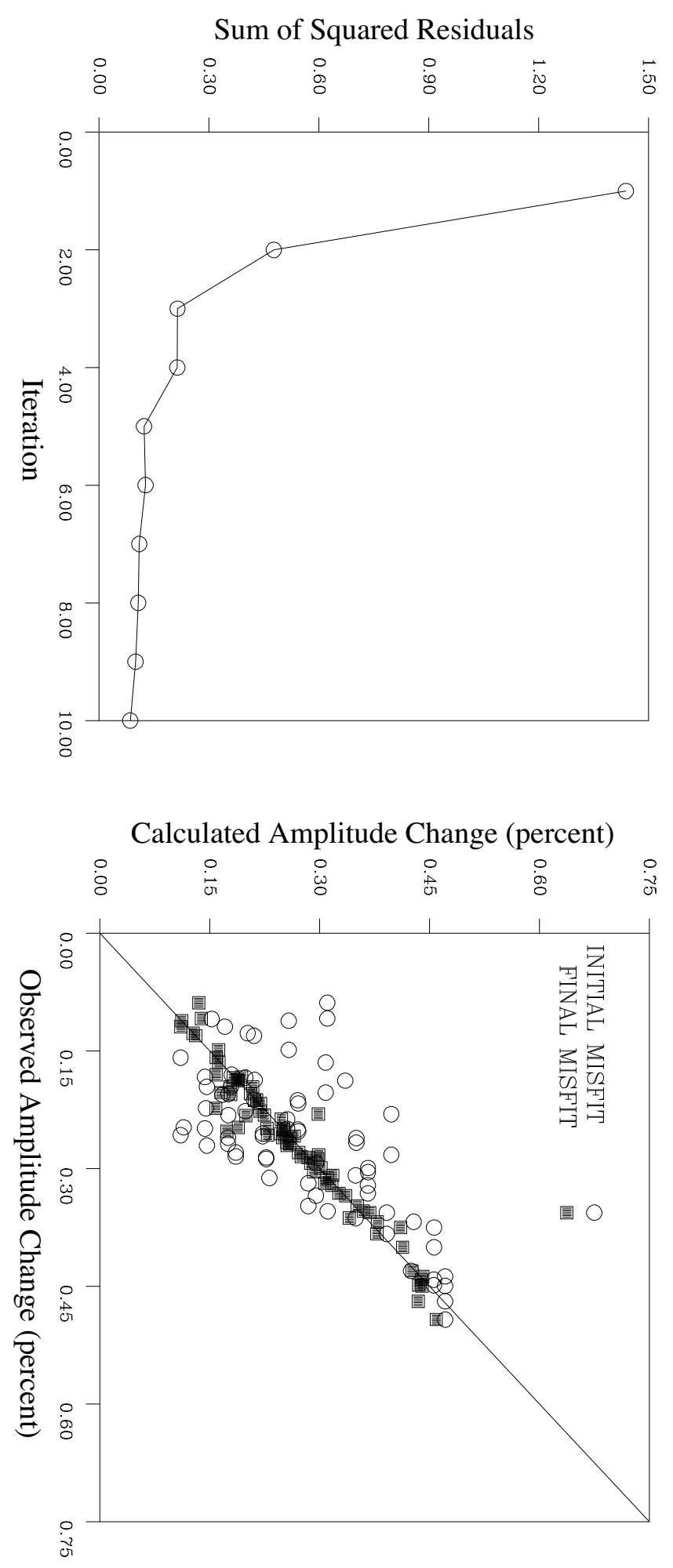

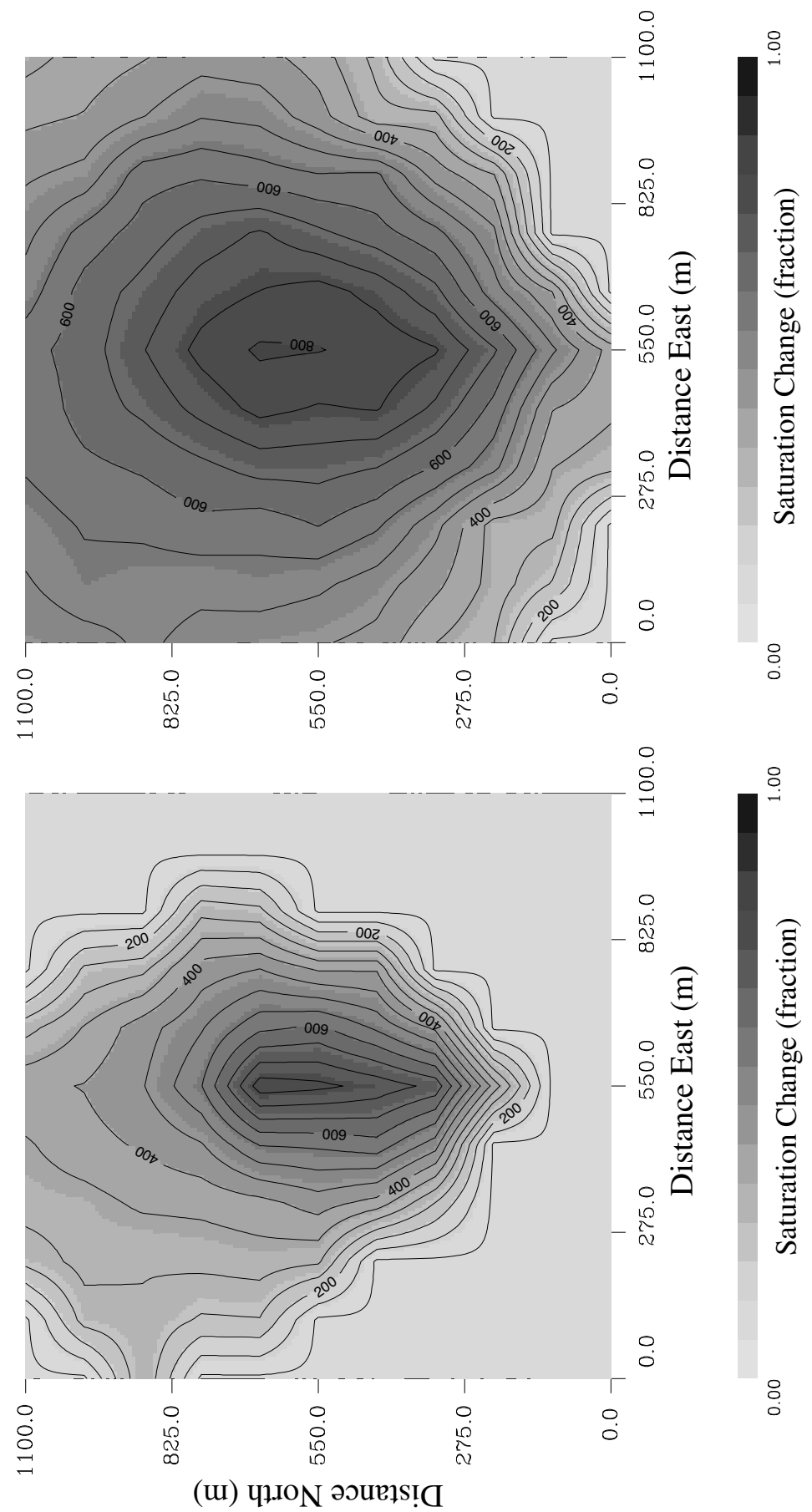


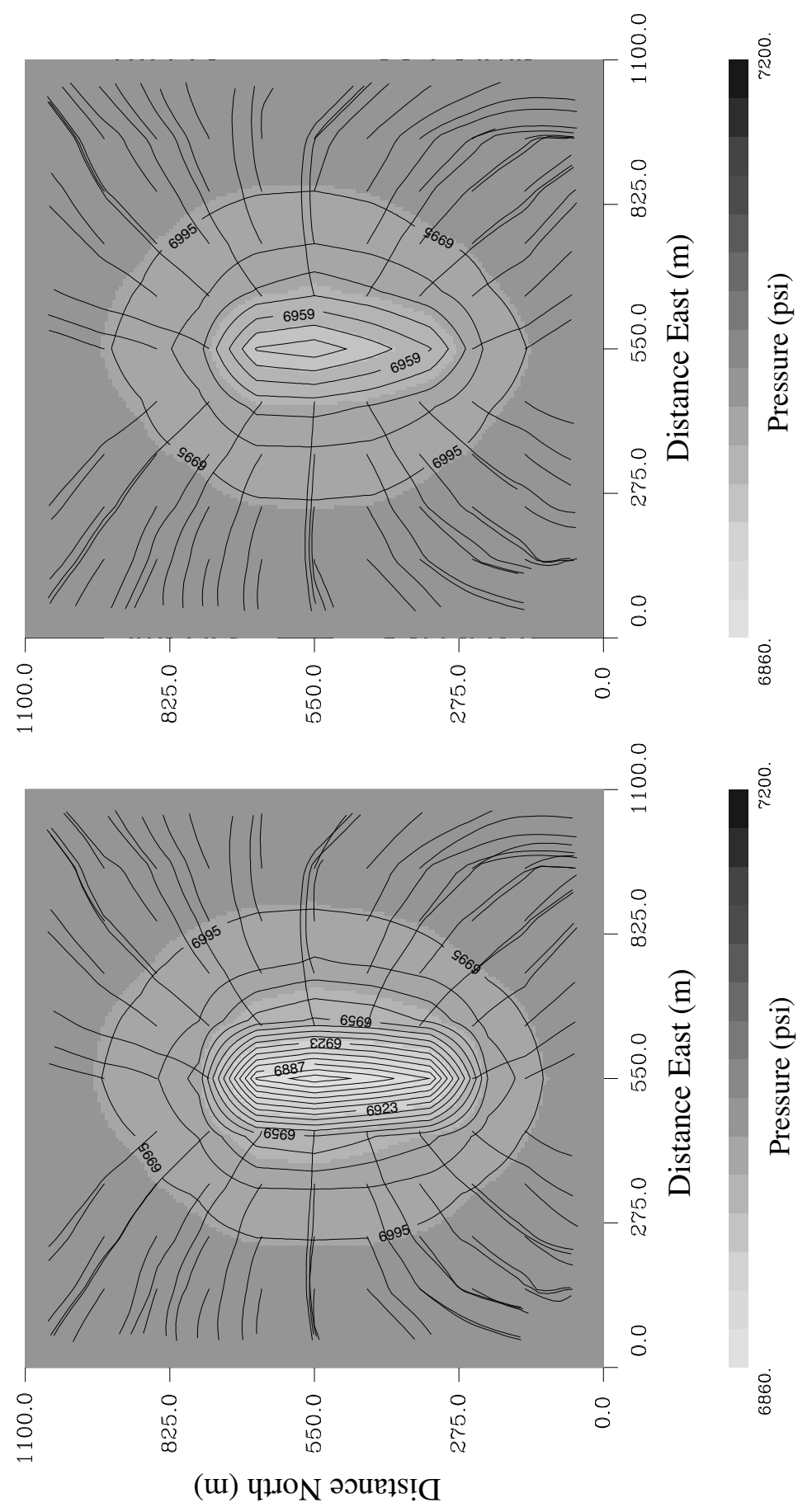



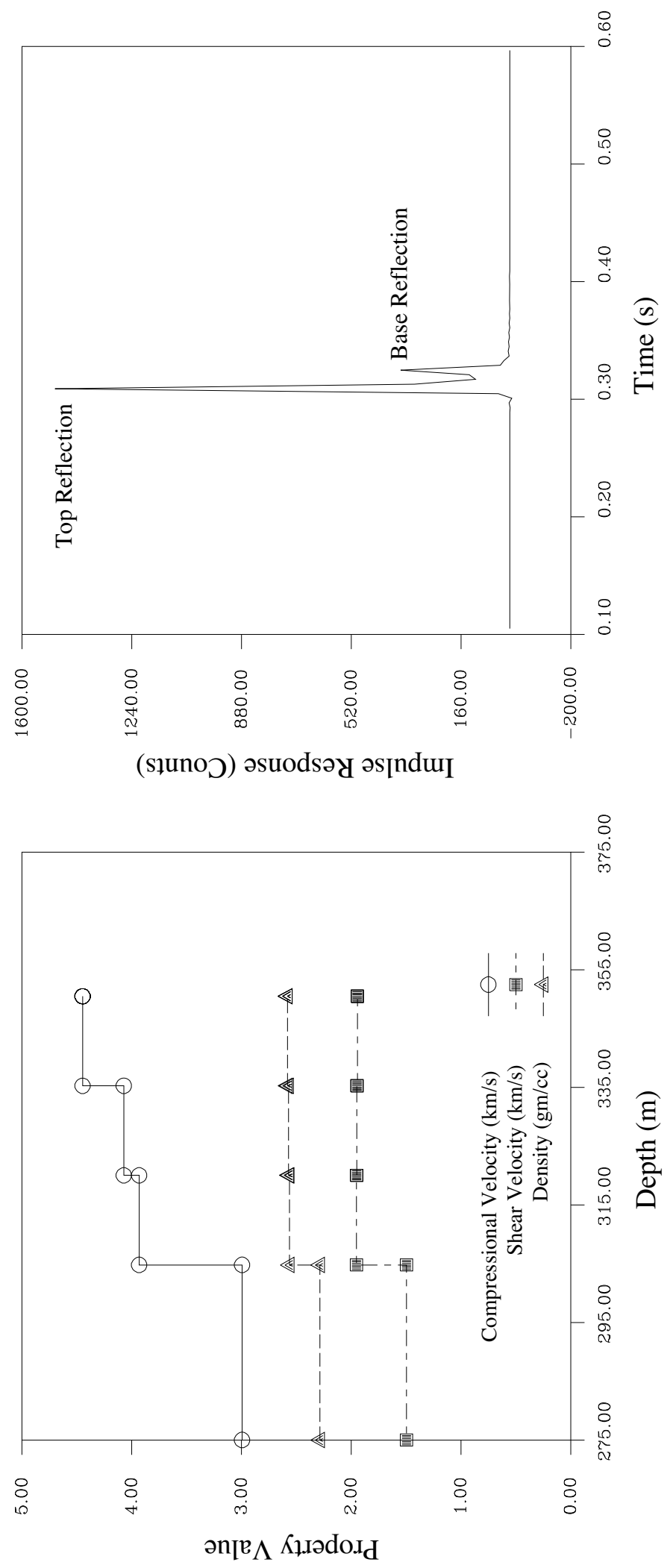


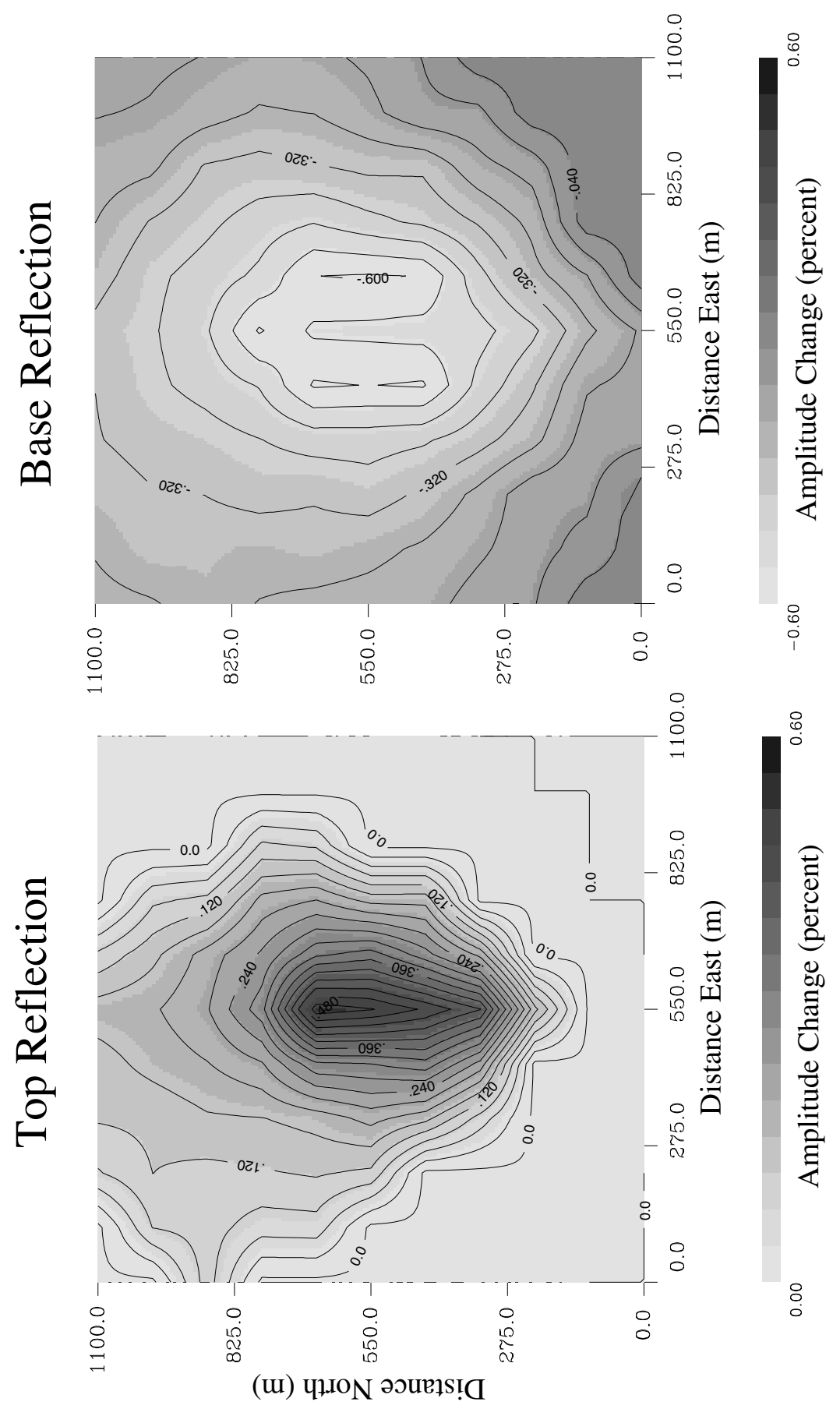




\section{Top Reflection}
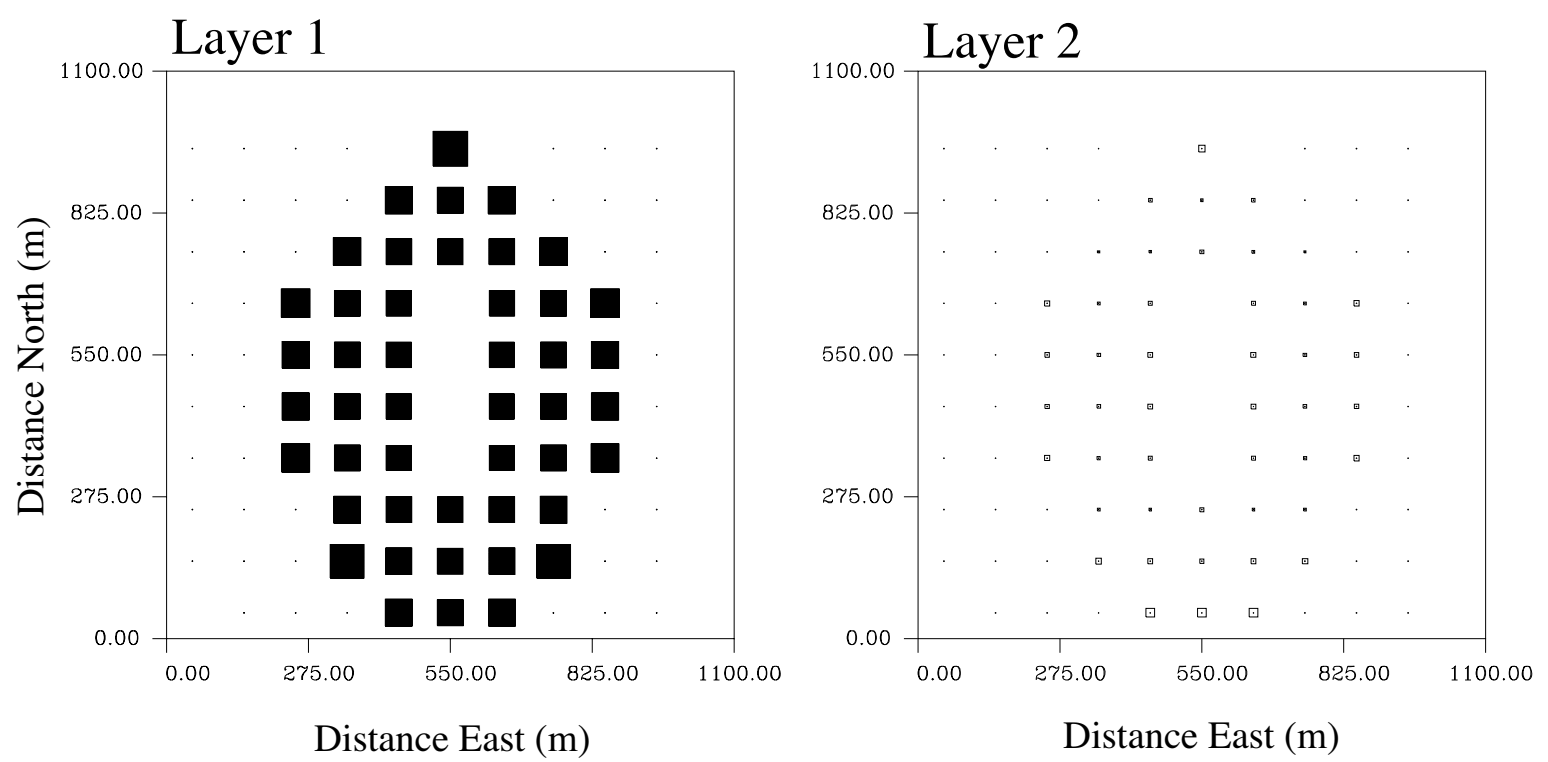

\section{Base Reflection}

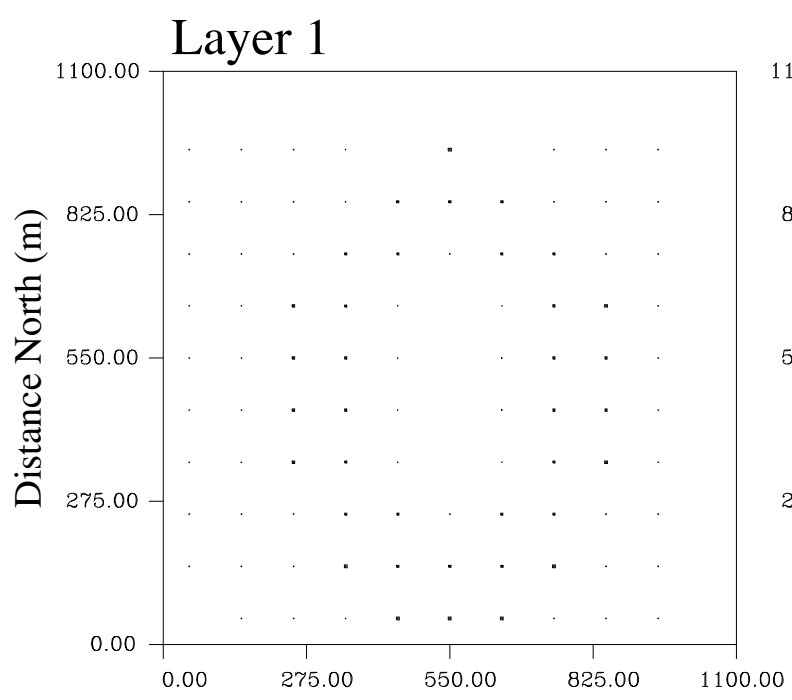

Distance East (m)

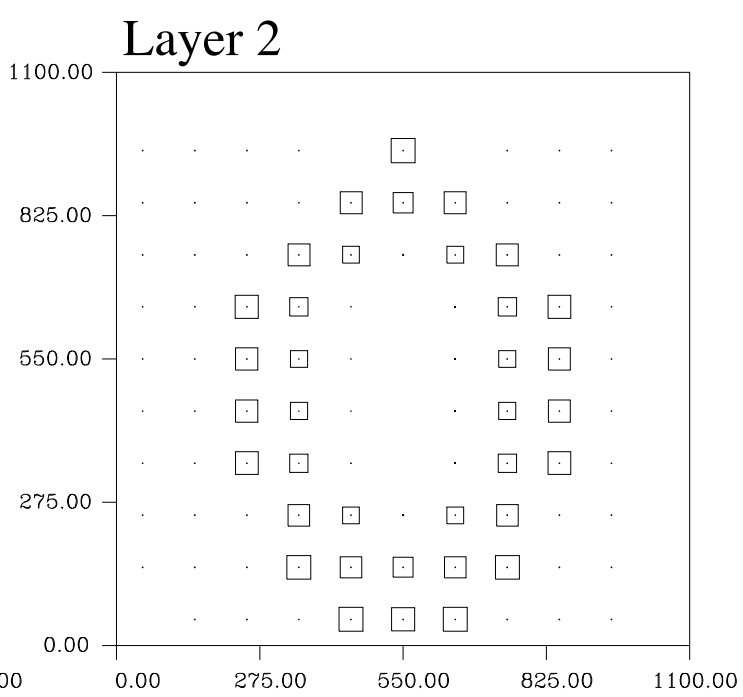

Distance East (m) 


\section{Top Reflection}
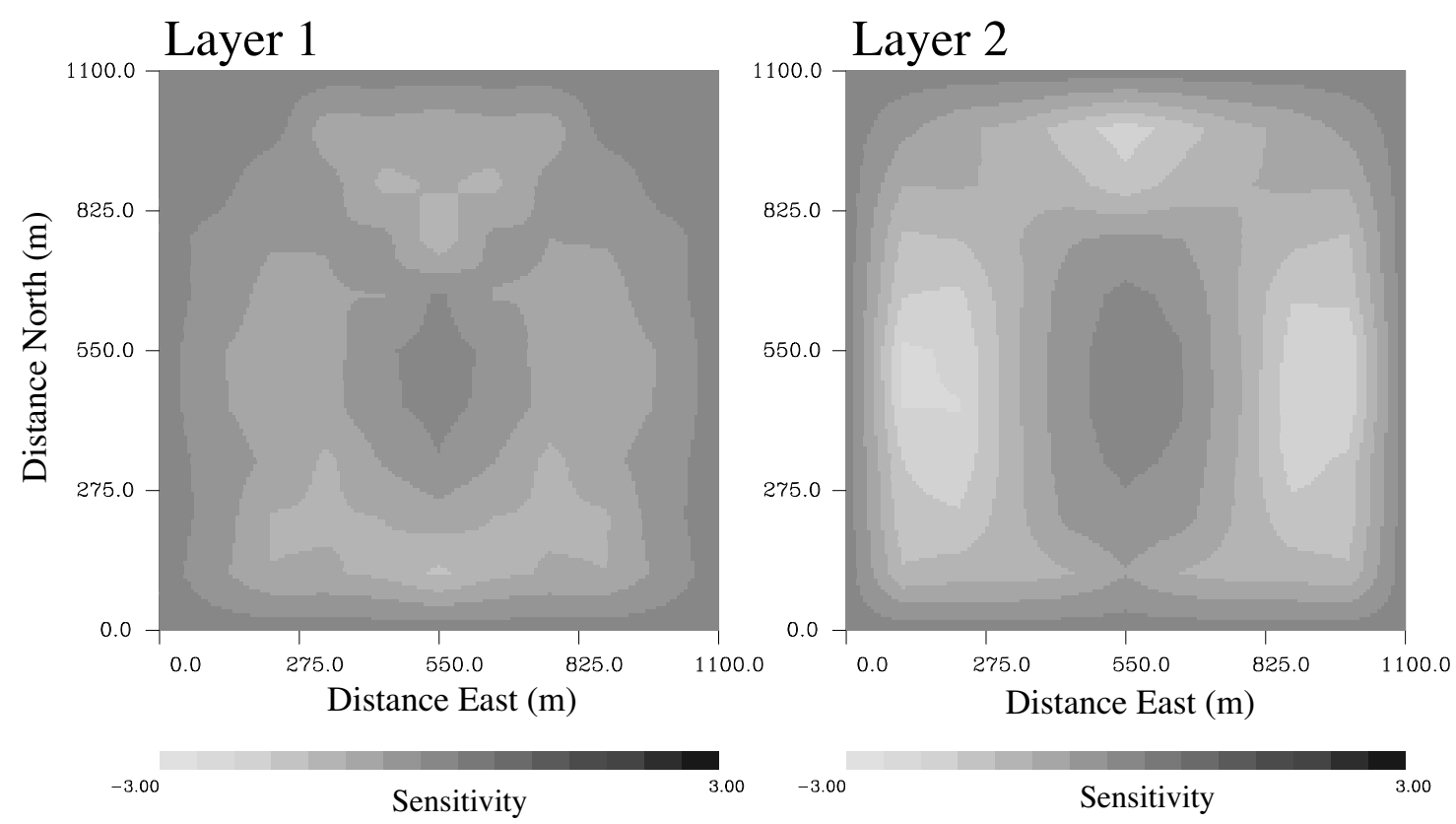

\section{Base Reflection}
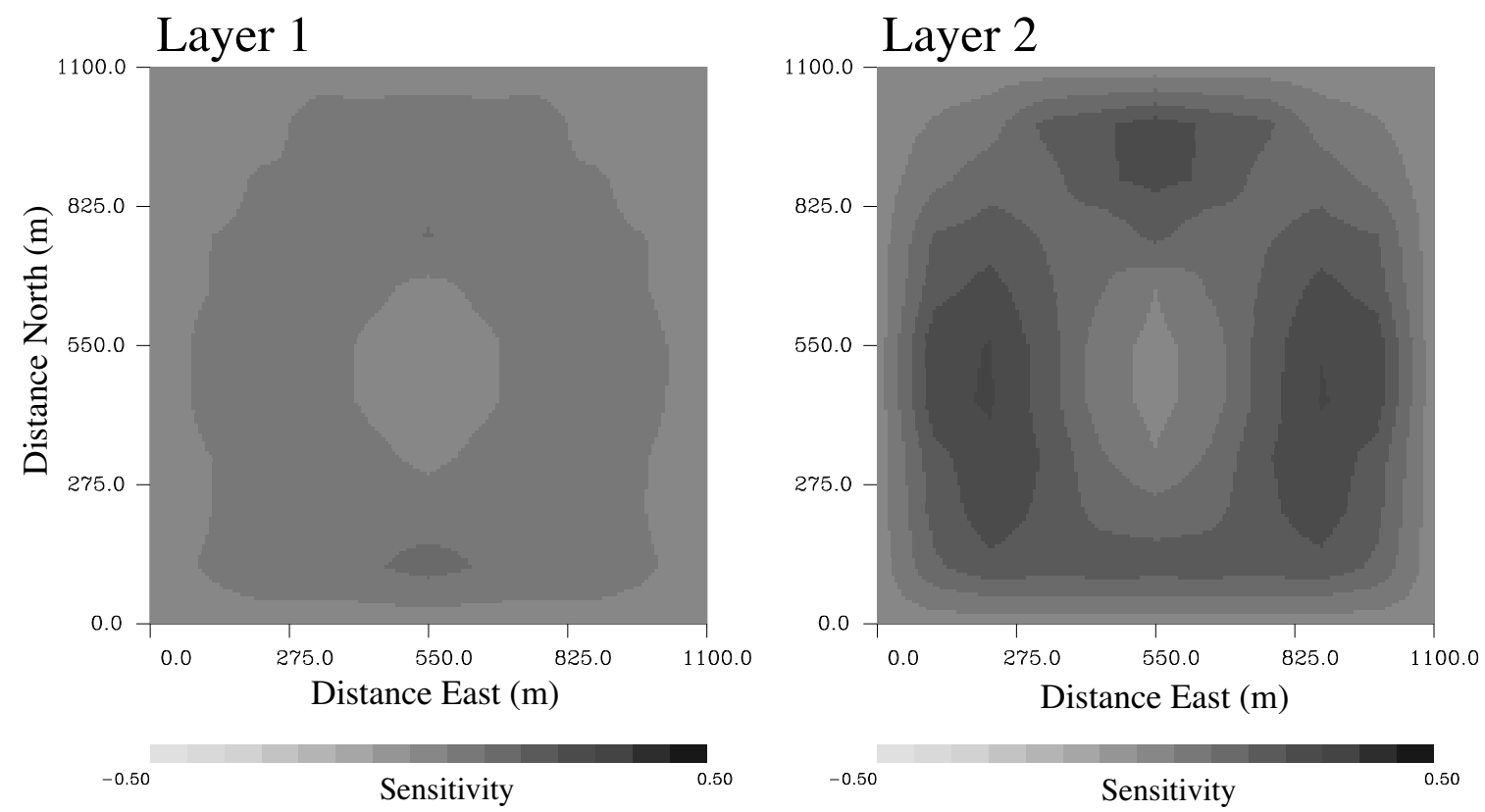


\section{Top Reflection}
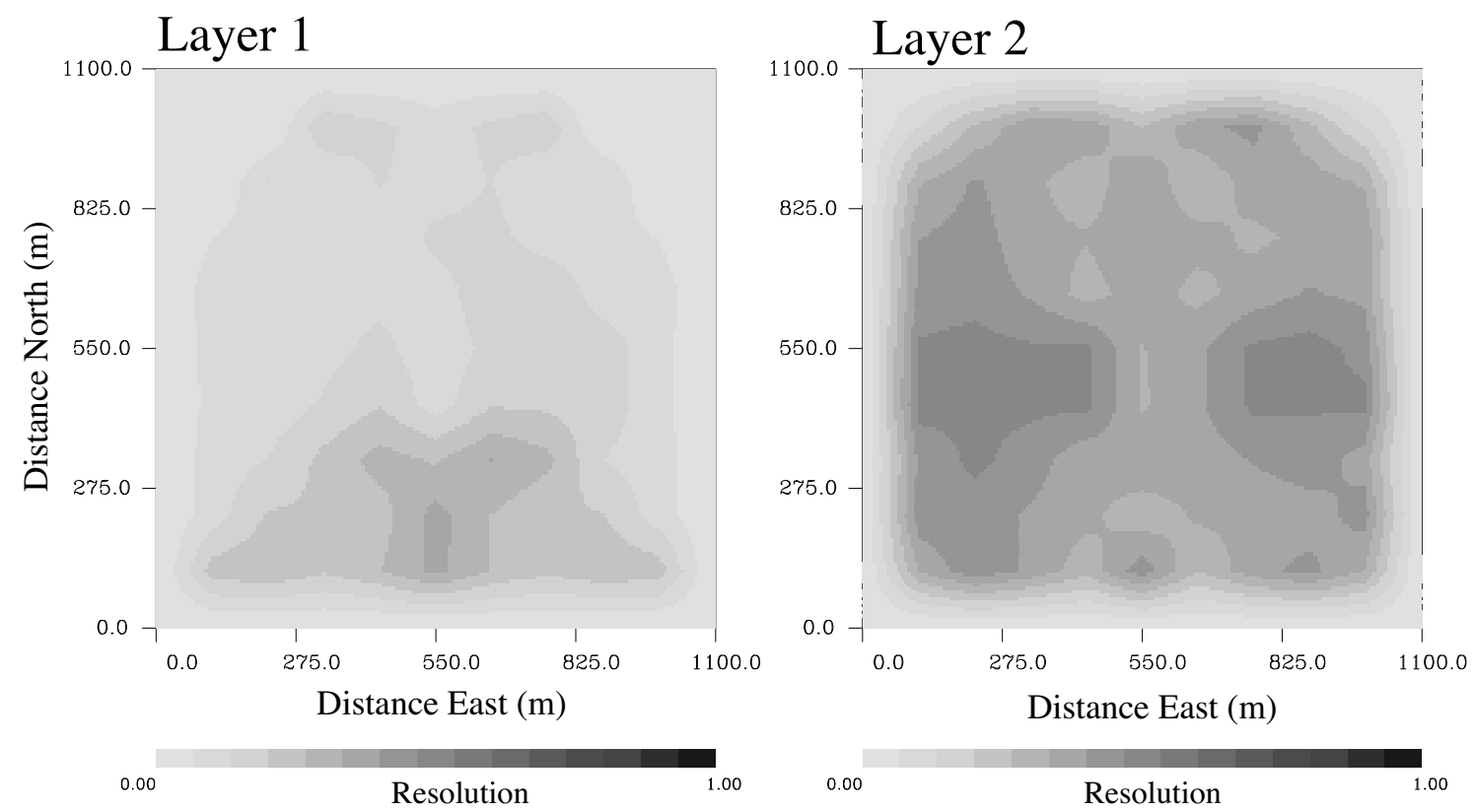

\section{Base Reflection}
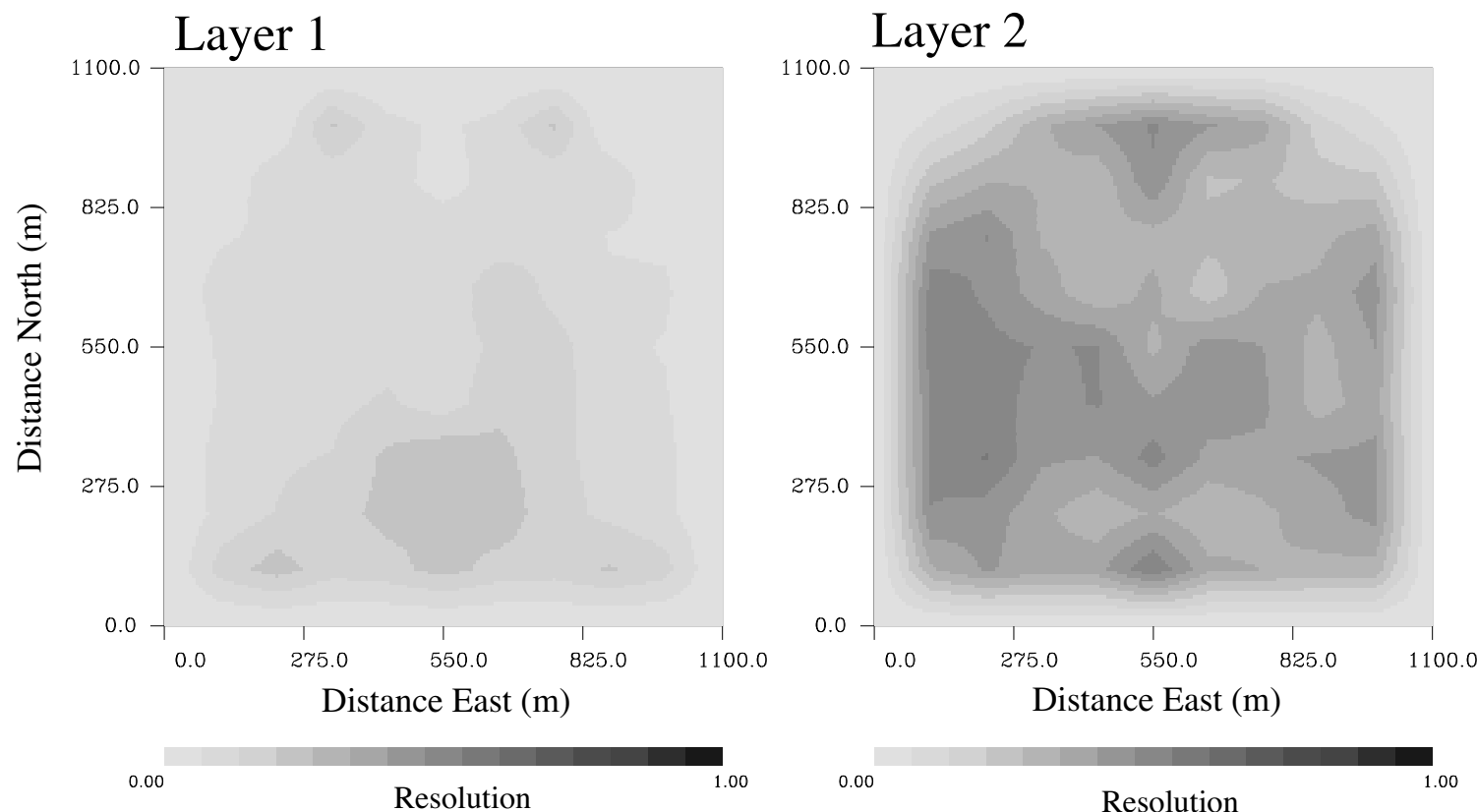


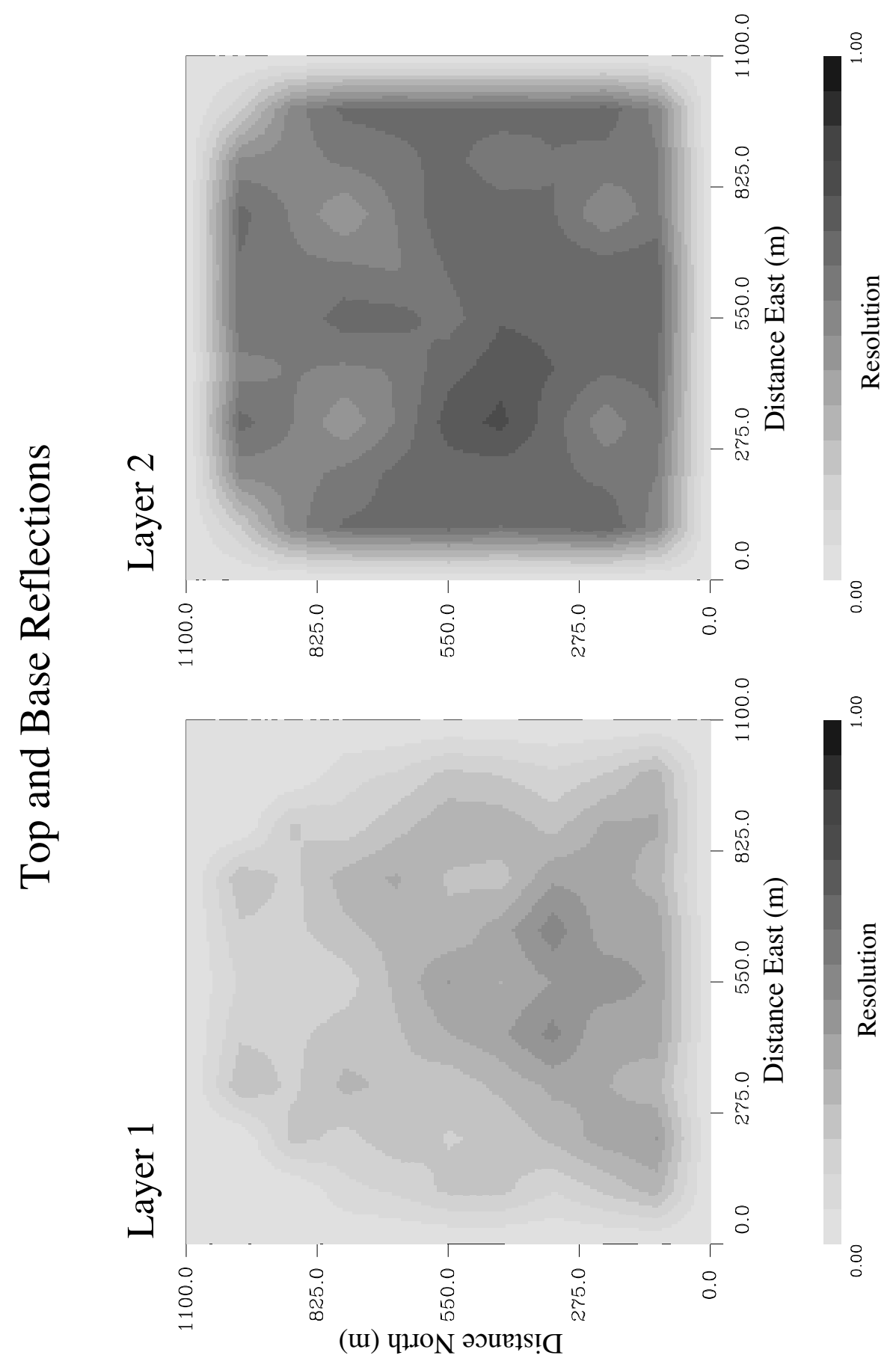




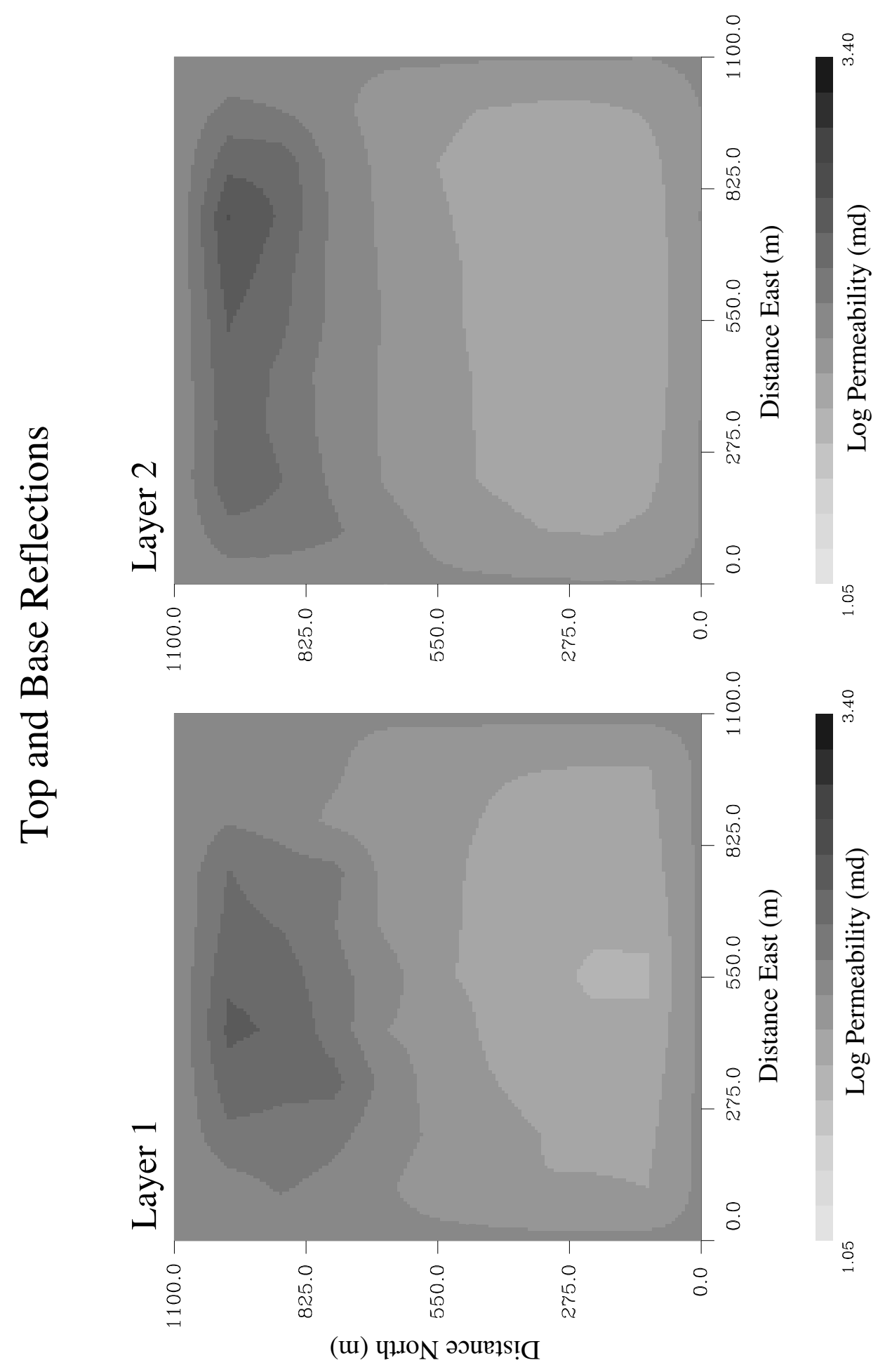




\section{Days}

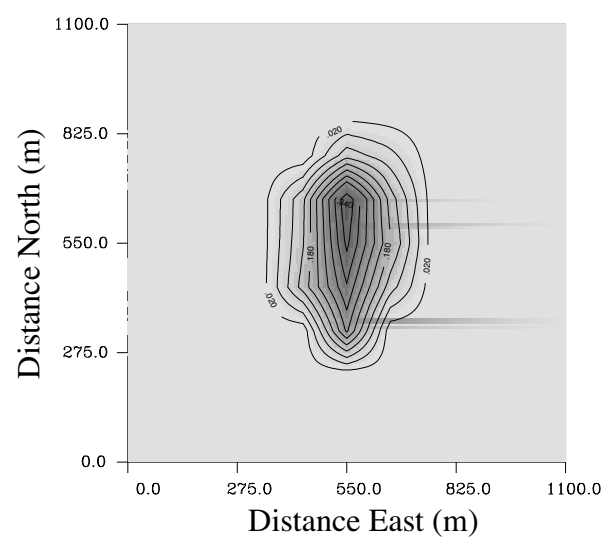

0.00 Amplitude Change (percent)

\section{Days}
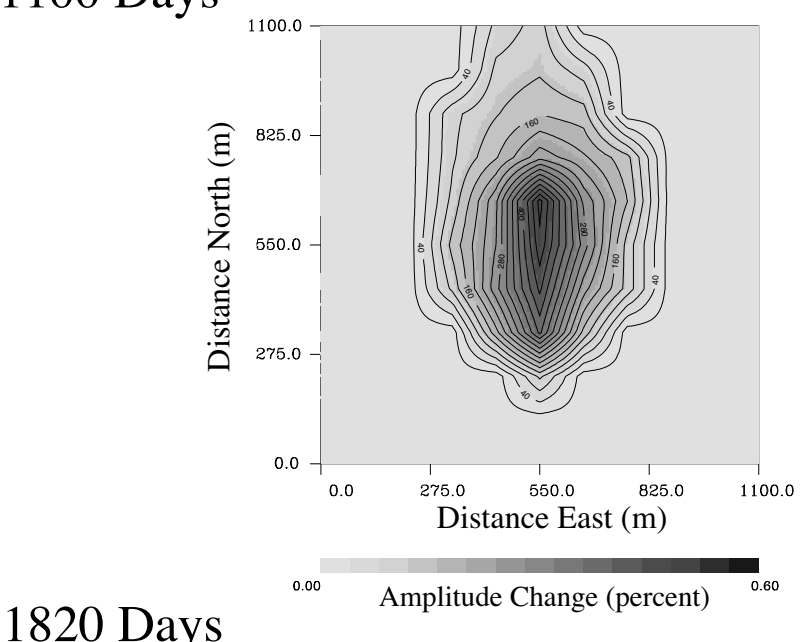

1820 Days

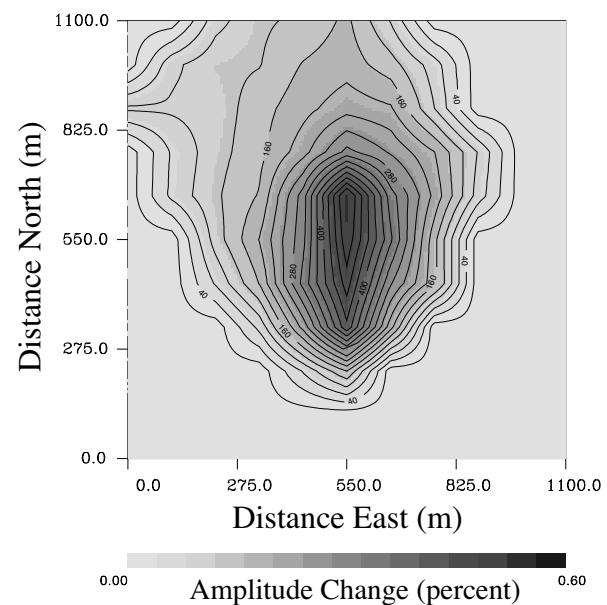




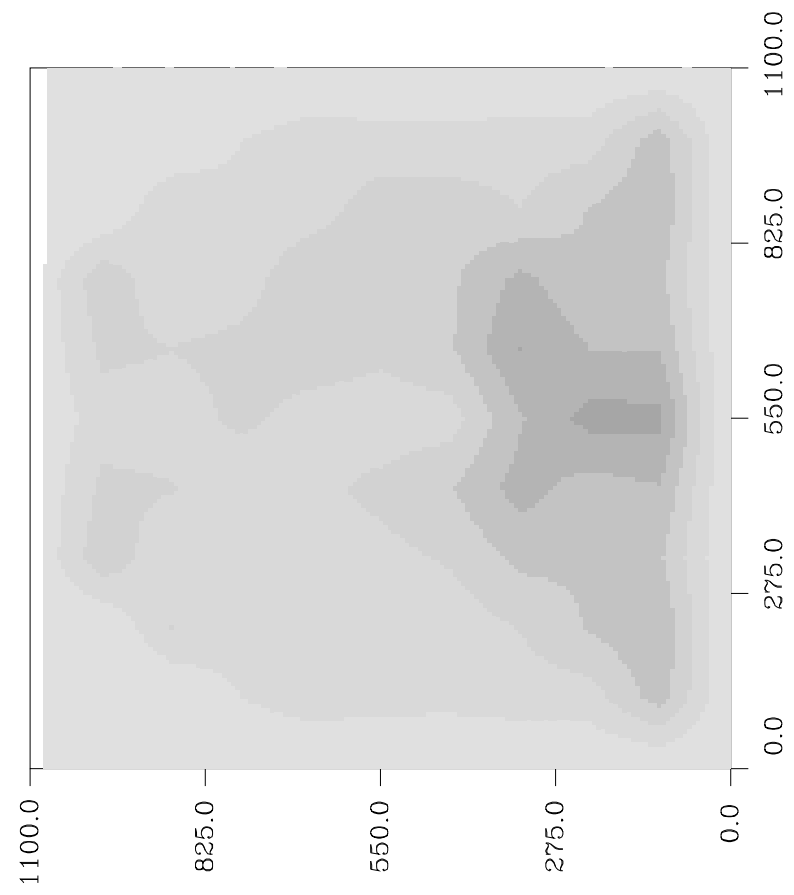





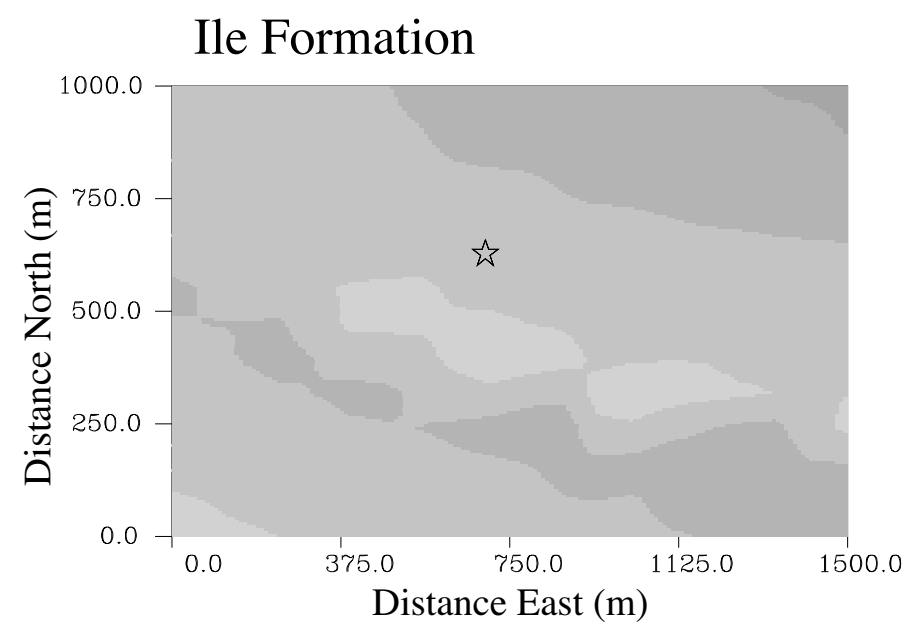

2400 .

Two-way-traveltime (ms)

2700.

\section{Tofte Formation}
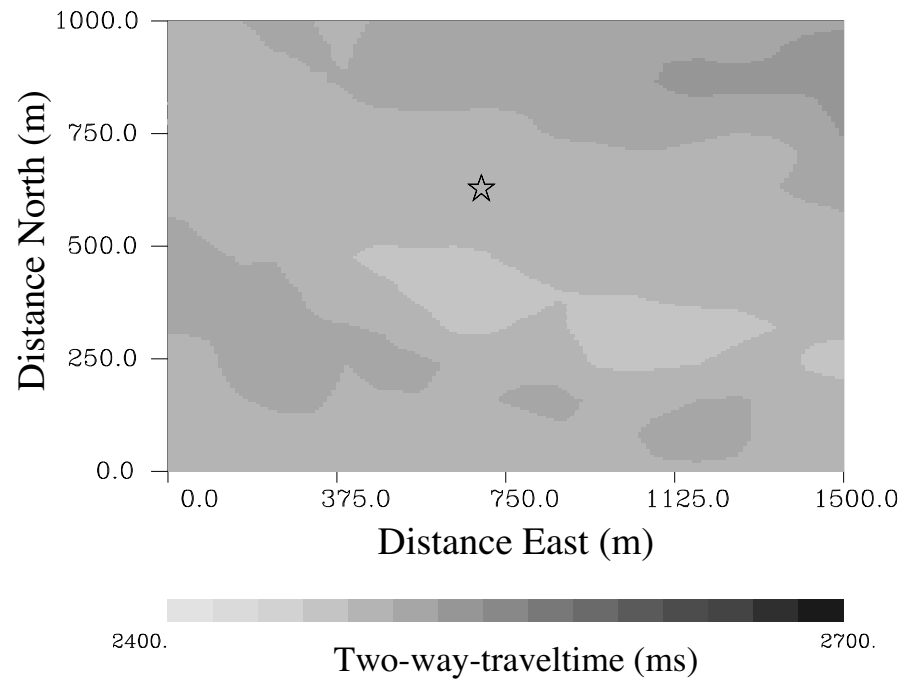

\section{Tilje Formation}

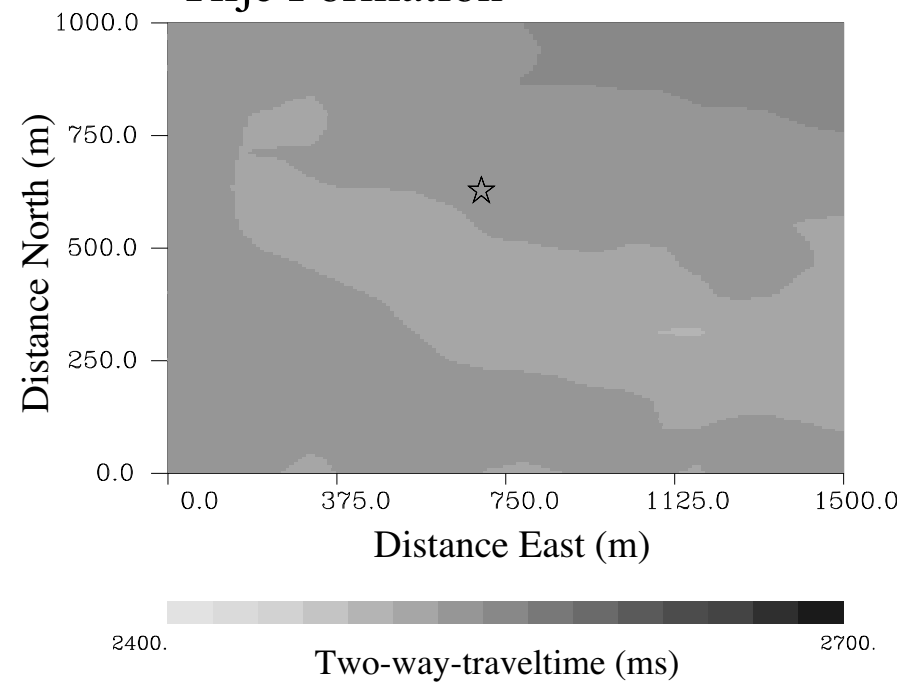





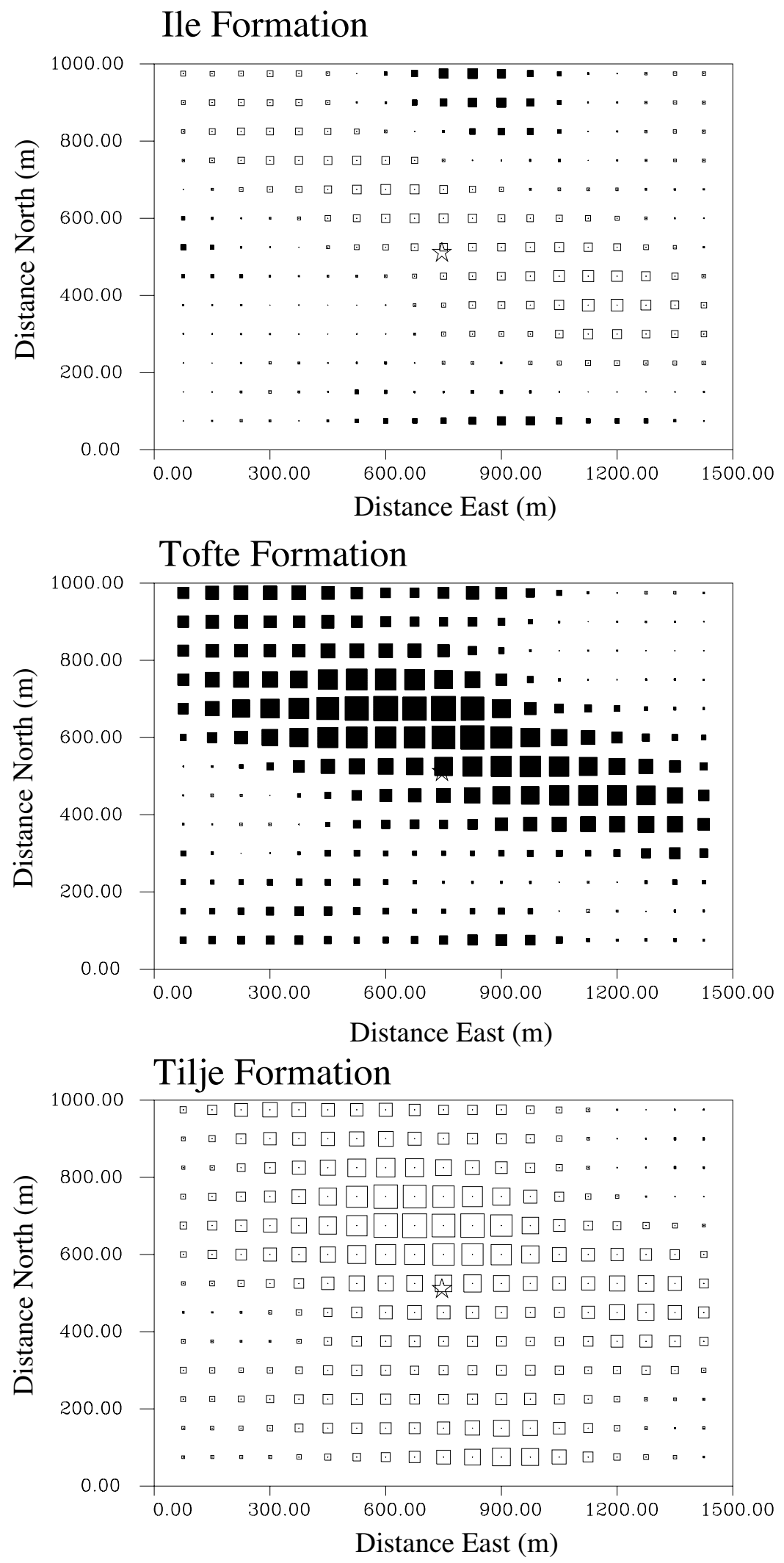


\section{Tofte Formation}

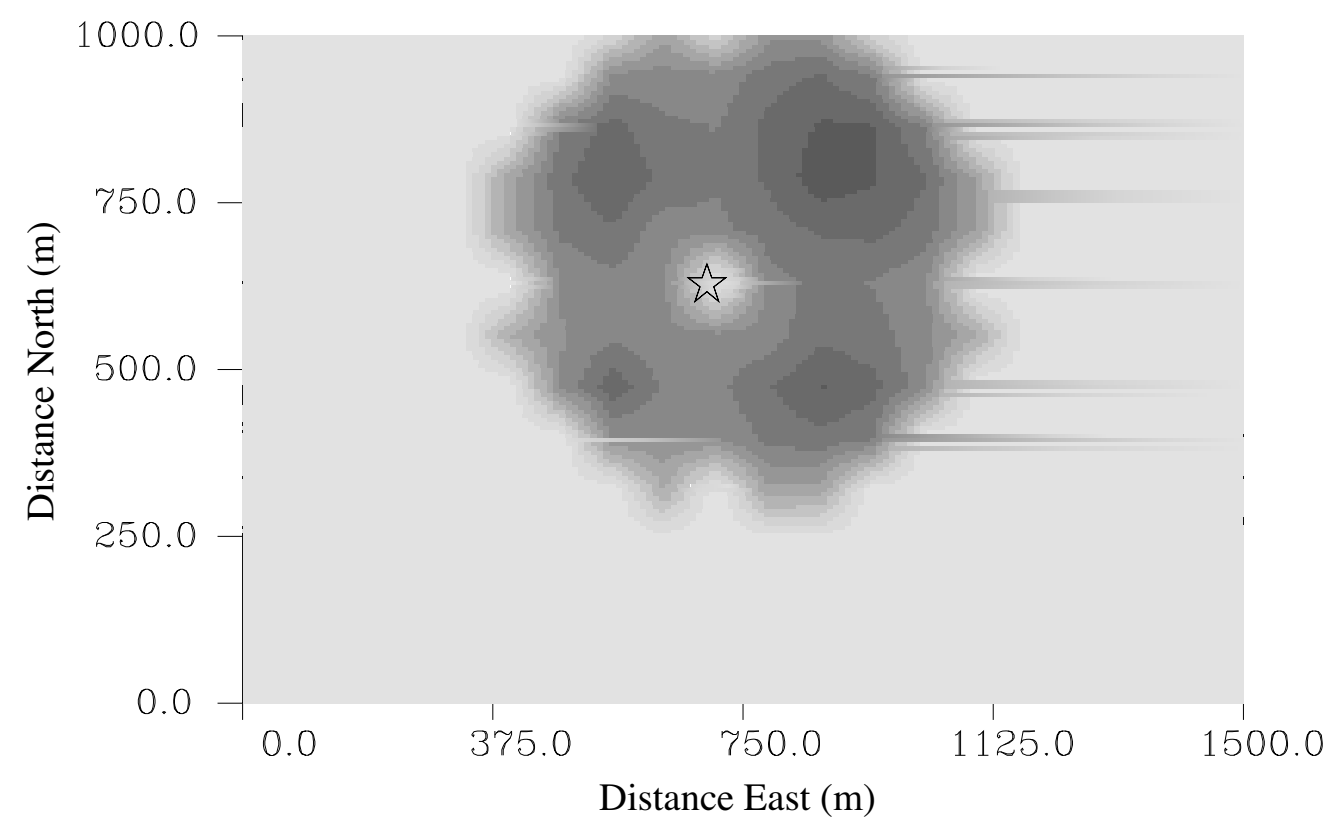

